\title{
Kinder-Mittelalterbilder als Möglichkeit und Herausforderung für Lehrende an Schulen
} Überlegungen aus der universitären Praxis

\section{Die Bedeutung des Mittelalters innerhalb und außerhalb von Bildungseinrichtungen}

„Weder im Geschichtsunterricht an den Schulen, aus dem das Mittelalter weithin verdrängt worden ist, noch bei der Mehrzahl der Studierenden des Fachs, die dem Zeitalter die Lateinsprachigkeit zum Vorwurf machen und es möglichst zu umgehen bzw. pflichtgemäß hinter sich zu bringen suchen [...], erfreut sich das Mittelalter einer besonderen Hochschätzung. " $\mathrm{Zu}$ dieser wenig hoffnungsvollen Einschätzung der Rolle der mittelalterlichen Geschichte kam Hans-Werner Goetz in seinem vor über 20 Jahren erschienenen Situationsbericht zu Stand und Perspektiven der Mittelalterforschung. In dem Jahr, in dem in Bologna europäische Verantwortliche der Bildungspolitik den Entschluss fassten, Studiengänge und -abschlüsse zu vereinheitlichen, die internationale Mobilität von Studierenden zu erhöhen und so einen europäischen Hochschulraum zu schaffen, konstatiert Goetz für Deutschland seit den 1970er Jahren steigende Studierendenzahlen bei sinkender Ausstattung der Mittelalter-Lehrstühle sowie deren Abbau. ${ }^{2}$ Damit beklagt er strukturelle Entscheidungen in Politik und Wissenschaft, die für die heutige Situation der mittelalterlichen Geschichte an Universitäten mutmaßlich verantwortlich sind.

Dass Veränderungen dieser Art die Lehre an Hochschulen beeinflussen, ist keine neue Entwicklung. Am Beispiel der Historischen Grundwissenschaften (oder Hilfswissenschaften), die für alle Epochen der Geschichtswissenschaft relevant sind, strukturell jedoch meistens mit Professuren für Mittelalterliche Geschichte verbunden sind, ist dies zu erkennen. Schon Ahasver von Brandt beklagte im Vorwort zu seinem Klassiker ,Werkzeug des Historikers` den Interessenwandel im Fach selbst, der zu einem Bedeutungsverlust der quellenkundlichen Grundwissenschaften geführt habe. ${ }^{3}$ Auch

\footnotetext{
1 Hans-Werner Goetz, Moderne Mediävistik. Stand und Perspektiven der Mittelalterforschung. Darmstadt 1999, 7.

2 Ebd., 142-145.

3 Ahasver von Brandt, Werkzeug des Historikers. Stuttgart 1958, 7.
}

\footnotetext{
>> Das Werk ist unter der Creative-Commons-Lizenz Namensnennung - Weitergabe unter gleichen Bedingungen 4.0 International veröffentlicht. Den Vertragstext finden Sie unter: https://creativecommons.org/licenses/by-sa/4.0/deed.de. Bitte beachten Sie, dass einzelne, entsprechend gekennzeichnete Teile des Werks von der genannten Lizenz ausgenommen sein bzw. anderen urheberrechtlichen Bedingungen unterliegen können.
} 
noch 55 Jahre später konstatiert Hiram Kümper in der Einleitung zu seiner Einführung in die Grundwissenschaften deren Bedeutungsverlust. ${ }^{4}$ Die mittelalterliche Geschichte an Hochschulen befinde sich nach diesen Ausführungen also in einer veritablen Dauerkrise, die durch Marginalisierung in den neueren Lehrplänen und Fachanforderungen noch verstärkt werde. Die mediävistische Geschichtswissenschaft könnte sich nun auf die methodengeleitete, quellengestützte Arbeit zurückziehen, Schullehre und ihre Rolle im Geflecht von (Hoch-)Schulbildung und Gesellschaft ignorieren und allein über Geschichtskultur in Museen in Kontakt mit einer interessierten Öffentlichkeit treten. Dagegen sprechen jedoch einerseits Beispiele für politisch-gesellschaftlichen Missbrauch von Mittelalter-Bildern zu nationalistischen oder geschichtsrevisionistischen Zwecken. ${ }^{5}$ Andererseits boomen Mittelalterbilder in der Geschichtskultur seit bald 40 Jahren: Dazu gehörten zwar auch Erfolge von großen Sonderausstellungen in Museen. ${ }^{6}$ Die meisten Produkte dieser Geschichtskultur sind jedoch als nicht-wissenschaftsnah einzuordnen, wie beispielsweise kommerziell erfolgreiche Kinofilme und Serien zeigen, ${ }^{7}$ welche ihrerseits auf teils ebenso erfolgreichen Buchvorlagen beruhen. ${ }^{8}$ Der Bereich analoger und digitaler Spiele wächst weiter. Inzwischen gehören Mittelalter-Märkte fest zum Repertoire lokaltouristischer Veranstaltungsformate ${ }^{9}$ und auch digitale Angebote, die keiner professionell-wissenschaftlichen Qualitätskontrolle unterliegen, scheinen dem ,echten ' Mittelalter den Rang abzulaufen. ${ }^{10}$

\section{Eindrücke zu den Mittelalterbildern an Schulen und Hochschulen}

Der wissenschaftliche Einfluss auf geschichtskulturelle Mittelalterbilder ist trotz vereinzelter Ausnahmen bescheiden: Personen der Bildungsforschung, Fachwissenschaft und Schulpraxis beklagen nämlich einhellig, dass sich diese Mittelalter-Begeisterung

\footnotetext{
4 Hiram Kümper, Materialwissenschaft Mediävistik. Einführung in die Historischen Hilfswissenschaften. Paderborn $2014,11$. 5 Vgl. dazu Fabian Link, Gegenwarten des Mittelalters vom späten 18. bis ins 20. Jahrhundert. Politisierung, Populärkultur und Kulturwissenschaften, in: GWU 67, 2016, 509-522; János M. Bak, Vorwort, in: Ders./ Jörg Jarnut/Pierre Monnet (Hrsg.), Gebrauch und Missbrauch des Mittelalters, 19.-21. Jahrhundert. München 2009 (MittelalterStudien, Bd. 17), 9-13; Chris Jones/ Conor Kostick/Klaus Oschema, Why Should we Care about the Middle Ages? Putting the Case for the Relevance of Studying Medieval Europe, in: Dies. (Hrsg.), How Medieval Studies Contribute to Improving our Understanding of the Present. Berlin/ Boston 2020 (Das Mittelalter, Beihefte 6), 9-11.

6 Eine Auswahl an überregional beworbenen Ausstellungen: Den Anfang machte, Die Zeit der Staufer. Geschichte - Kunst Kultur' in Stuttgart 1977 anlässlich des 25. Geburtstags Baden-Württembergs; 1992 in Speyer ,Das Reich der Salier 1024-1125'; in Magdeburg 2001 ,Otto der Große, Magdeburg und Europa'; sowie die Doppelausstellungen ,Burg und Herrschaft' (Deutsches Historisches Museum, Berlin) und ,Mythos Burg' (Germanisches Nationalmuseum, Nürnberg) 2010.

7 Vgl. z. B. Der Name der Rose (D/F/I 1986, Regie: Jean-Jacques Annaud); Kingdom of Heaven (USA/UK/D/E/MAR 2005); die Welt von ,Game of Thrones' (USA 2011-19, nach ,A Song of Ice and Fire' von George R.R. Martin 1996-2011, zwei Bände sind noch angekündigt) ist zwar fiktiv, aber am europäischen Mittelalter orientiert.

8 Vgl. z. B., Der Name der Rose' von Umberto Eco 1980 (D 1982); Ken Follets, Die Säulen der Erde' (UK 1989/ D 1992; Verfilmung (CA/D/HUN/UK 2010); , Die Wanderhure' von Iny Lorentz 2004 (Verfilmung D/AT 2010.)

9 Eine Informationsseite mit einer Übersicht für Deutschland bietet https://www. mittelalterkalender.info (letzter Zugriff: 26.5.2021).

10 Die Gefahr benennen Jones /Kostick/Oschema, Why Should we Care (wie Anm. 5), 3.
} 
nicht im Wissen der Schüler*innen niederschlage. ${ }^{11}$ Dabei werden bezeichnenderweise kaum Kompetenzen thematisiert. Ein Wissenskanon ist schnell formuliert, doch transportiert Geschichtskultur offenbar weder Wissen noch Kompetenzen zu Mittelalterbildern oder diese werden nicht rezipiert. Ähnliche Erfahrungen kursieren unter Lehrenden beim informellen Austausch: Die Lehrperson glaubt inhaltlich, methodisch und didaktisch alle Register gezogen zu haben und doch scheint dies - in Anbetracht der Prüfungsergebnisse - für manche Studierende in einem Paralleluniversum oder einer Paralleluniversität stattgefunden zu haben. Hochschullehrende und Lehrkräfte an Schulen tradieren sprichwörtliche Klagen über die ,Jugend von heute also weiterhin. Im Gegensatz zu Filmen und Romanen ist es der Zweck von Lehre an Hochschulen, sowohl Wissen und Kompetenzen im Bereich der Geschichte zu vermitteln. Studien über den tatsächlichen Erfolg oder Misserfolg von Hochschullehre zur mittelalterlichen Geschichte in Bezug auf Schulen liegen nicht vor, abgesehen von der deutlichen Kritik Thomas Martin Bucks an der Geschichtswissenschaft. Diese habe zwar die mangelnde Vermittlung mittelalterlicher Geschichte an Schulen kritisiert, aber keinen Beitrag zur Umsetzung neuer Lehrpläne und damit zur Erneuerung des Mittelalterunterrichts geleistet. ${ }^{12}$ Mittels Einzelevaluationen von Lehrveranstaltungen und der nicht sonderlich aussagekräftigen Nennung von Examenszahlen, also den verbreitetsten Maßnahmen zur Qualitätssicherung an Universitäten, ist der Transfer von Wissen und Kompetenz von Universitäten an Schulen zu den Schüler*innen nicht zu erfassen. Zudem fühlen sich manche Studierende besser durch YouTubeFormate als durch besagte Lehre informiert, wie Kommentare zu ,musstewissen Geschichte - Leben im Mittelalter ${ }^{\star}$ - inzwischen MrWissen2go Geschichte - zeigen. ${ }^{13}$ Auch manche Lernende geben solchen Formaten den Vorzug vor dem Schulunterricht, sodass einzelne Lehrpersonen scheinbar gar nicht mehr selbst unterrichten. ${ }^{14}$ Die öffentliche Forderung nach einer Deakademisierung der Lehrer*innenbildung ${ }^{15}$ will zwar gewiss nicht YouTube den Schulunterricht überlassen. Doch die geschichtswissenschaftliche Mediävistik trägt zu diesem Diskurs in Bezug auf Veröffentlichungen

11 Nicola Brauch, Die vergessenen Fragen an die mittelalterliche Geschichte, in: Johannes Grabmayer (Hrsg.) Das Bild vom Mittelalter. Klagenfurt 2013 (Schriftenreihe der Akademie Friesach. Neue Folge 3), 169-198, hier 169.

12 Thomas Martin Buck, Mittelalter und Moderne. Plädoyer für eine qualitative Erneuerung des Mittelalter-Unterrichts an der Schule. 2. Aufl. Schwalbach 2014 (Forum Historisches Lernen), 41.

13 Mirko Drotschmann ist Journalist und hat Geschichte studiert. Video zur Ständegesellschaft - Mittelalter https://www. youtube.com/watch?v=BRXQvm-tG6U (letzter Zugriff: 26.6.2021), Kommentar von dem Usernamen hanna eckart: „Du rettest mein Grundwissen fürs Studium!!"

14 Zu demselben Video der Username Winchester rifle „Wieder mal richtig gut erklärt, sehr anschaulich gestaltet und nicht so langweilig beim zuhören (nicht so wie in der Schule:-))" und Queen of Madita: „Unser lehrer macht kein Unterricht mehr er zeigt uns einfach am smart board die videos".

15 Mathias Brodkorb, Katja Koch, Klaus Zierer vertreten in Die Zeit Nr. 52/2019 die These, dass Universitäten Lehramtsstudierende zu Wissenschaftler*innen machen wollten: https://www.zeit.de/2019/52/lehrer-beamtenstatus-ausbildung-akademie-unterricht (letzter Zugriff: 26.5.2021). 
eher im Gefolge der Didaktik bei, ${ }^{16}$ obwohl zum Thema Schule und Bildung nahezu jeder Mensch in Deutschland eine Meinung hat, wie kürzlich in der ARD-Themenwoche zu erfahren war. ${ }^{17}$ Im Zuge der Corona-Pandemie gelangt dazu auch die Rolle der Digitalisierung in den Fokus, die breit in der Gesellschaft diskutiert wird. Manche der Initiator*innen wollen dabei u. a. „die Lehrpläne entrümpeln“. ${ }^{18}$ Bei solchen Diskussionen steht das Fach Geschichte nicht im Zentrum des Interesses - und professionelles Wissen und Kompetenzen bezüglich aktueller, kompetenzorientierter Lehrpläne sind bei Diskutant*innen eher nicht zu erschließen. Das Bild vom verzichtbaren „alten Wissen [...] viele[r] [...] Jahreszahlen in Geschichte“ spukt immer noch in den Köpfen herum, ${ }^{19}$ obwohl in den Fachanforderungen kaum noch Jahreszahlen stehen. Buck fordert in seiner Studie für den Mittelalterunterricht vielmehr eine rezeptions- und wirkungsgeschichtliche Ausrichtung, um einen Bezug zur Lebenswelt der Schüler*innen herzustellen. ${ }^{20}$ Außerdem regt er eine stärkere Kooperation zwischen fachwissenschaftlicher und fachdidaktischer Wissenschaft sowie den ausführenden Schulen an. ${ }^{21}$

\section{Forschungsstand zu rezeptionsgeschichtlichen Perspektiven verschiedener Fächer}

Im Bereich ,Film' steht der breiteste Forschungsstand zur Verfügung. ${ }^{22}$ Zum Thema ,Dokumentation' liegt ein zeitgeschichtlicher Schwerpunkt mit der Diskussion von Aspekten wie Authentizität, Geschichtsbildern und dem Erlernen historischer Kompe16 Auf den Fluren und unter den Lehrenden der Mittelalter-Abteilungen wird mitunter ein intensiver Austausch über Hochschul-
lehre gepflegt, der aber selten zu Veröffentlichungen führt. Impulse gehen von Zusammenarbeiten von Geschichtsdidaktik und
Geschichtswissenschaft aus: Thomas Martin Buck/Nicola Brauch (Hrsg.), Das Mittelalter zwischen Vorstellung und Wirklichkeit.
Probleme, Perspektiven und Anstöße für die Unterrichtspraxis, Münster 2011; als Beispiel für die Umsetzung in der Lehre die Zu-
sammenarbeit von Andreas Bihrer und Nicola Brauch bei,FOGEL - Forschende Geschichtslehrer_innen', Lehramtsausbildung an der
Schnittstelle von Wissenschaft \& Didaktik. Hierzu der Artikel vom 2. Juli 2015 im Lehre-Blog der CAU Kiel http://www.einfachgu-
telehre.uni-kiel.de/allgemein/fogel/ (letzter Zugriff: 26.5.2021); noch mit einem stark fachwissenschaftlichen Schwerpunkt: Rolf
Ballof (Hrsg.), Geschichte des Mittelalters für unsere Zeit. Erträge des Kongresses des Verbandes der Geschichtslehrer Deutsch-
lands „Geschichte des Mittelalters im Geschichtsunterricht“ Quedlinburg 20.-23. Oktober 1999. Wiesbaden/Stuttgart 2003 .

17 ARD-Themenwoche, Zukunft Bildung', 9.-16.11.2019.

18 Z. B. Der Hackathon ,\#wirfürschule' (8.6.-12.6.2020), der vom Bundesministerium für Bildung und Forschung unterstützt wurde, https://www.bildung-forschung.digital/de/wirfuerschule---der-hackathon-fuer-die-zukunftsschule-3120.html (letzter Zugriff: 26.5.2021). Steve Haak zitiert die beiden Initiator*innen und Gründer*innen Verena Pausders (Digitale Bildung für alle e.V.) und Michael Maendler (www.lehrermarktplatz.de) mit diesen Worten im Beitrag ,Hackathon-Initiator: „Wir wollen die Lehrpläne entrümpeln"' bei Business Insider (9.6.2020), https://www.gruenderszene.de/technologie/hackathon-wirfuerschule?interstitial (letzter Zugriff: 26.6.2021).

19 Franz Fehrenbach, Vorsitzender des Aufsichtsrats der Robert Bosch GmbH, in einem Interview zum Umbau von Schulen in Die Zeit 29 (12.7.2018): https://www.zeit.de/2018/29/bildung-reform-deutschland-schulen-lehrer (letzter Zugriff: 26.6.2021).

20 Buck, Mittelalter (wie Anm. 12), $81 \mathrm{f}$.

21 Ebd., 41.

22 Zu den Einsatzmöglichkeiten von Filmen in der Hochschullehre vgl. Janina Lillge, Spielfilme in der geschichtswissenschaftlichen Lehre. Chancen, Herausforderungen und Möglichkeiten, in: Martin Fischer/ Michaela Pölzl (Hrsg.), Blockbuster Mittelalter. Akten der Nachwuchstagung Bamberg. 11.-13.06.2015. Bamberg 2018, 489-517, mit einer Übersicht zur Literatur mit Mittelalterschwerpunkt. 
tenzen vor. ${ }^{23}$ Diese Ergebnisse sind nur bedingt auf mittelalterliche Beispiele übertragbar, da weder zeitgenössisches Filmmaterial vorliegt noch Zeitzeug*inneninterviews möglich sind.

Im englischsprachigen Raum sind interdisziplinäre Studien zu rezeptionsgeschichtlichen Perspektiven unter der Forschungsrichtung ,Medievalism‘ stärker als im deutschsprachigen Raum verbreitet, wo diese eher in Literaturwissenschaften und in der Geschichtswissenschaft in Ringvorlesungen eine Rolle spielen. ${ }^{24}$

Andere Untersuchungen und Analysen gingen meistens aus universitärer Lehre oder dem Bereich der Geschichtsdidaktik hervor. ${ }^{25}$ Abgesehen von den Analysen der Geschichtsdidaktik $^{26}$ fokussieren die Untersuchungen zumeist implizit Erwachsene, obwohl die Frage nach dem Interesse von Erwachsenen und Lehrenden am Mittelalter jüngst erst fokussiert wird. ${ }^{27} \mathrm{Zu}$ den besonderen Voraussetzungen bei Studien mit und zu Kindern im Bereich der Geschichtskultur hat sich vor allem die Medienforschung profiliert. ${ }^{28}$ Die Pädagogik und Geschichtsdidaktik folgten nämlich lange der Ansicht der 1950er und 60er Jahre, dass Schüler*innen der Grundschule nicht die entwicklungspsychologische Reife für historisches Lernen besäßen. ${ }^{29}$ Damit ist die geringe Repräsentanz des Historischen Lernens in Grundschullehrplänen zu erklären, die nicht

23 Beispielsweise Thomas Fischer, Alles authentisch? Popularisierung der Geschichte im Fernsehen. Konstanz 2008; Sarah Böhlau, Diener zweier Herren. Der Mittelalterdokumentarfilm zwischen Authentizitätsanspruch und Unterhaltungswunsch, in: Fischer/Pölzl (Hrsg.), Blockbuster (wie Anm. 22) 519-528; Bodo von Borries, Geschichtslernen am Fernsehschirm. Analysemethoden, Fallbeispiele, Praxiserfahrungen, in: Hans-Jürgen Pandel/Gerhard Schneider (Hrsg.), Handbuch Medien im Geschichtsunterricht. Düsseldorf 1985 (Geschichtsdidaktik. Studien, Materialien, Bd. 24), 537-555; Christian Huck, Authentizität im Dokumentarfilm. Das Prinzip des falschen Umkehrschlusses als Erzählstrategie zur Beglaubigung massenmedialen Wissens, in: Antonius Weixler (Hrsg.), Authentisches Erzählen. Produktion, Narration, Rezeption. Berlin 2012 (Narratologia, Bd. 33), 239-264.

24 So ist dies auch beim International Medieval Congress, der jährlich in Leeds stattfindet, einer der vorgeschlagenen Untersuchungsaspekte. Vgl. Mathias Herweg/Stefan Keppler-Tasaki (Hrsg.), Rezeptionskulturen. Fünfhundert Jahre literarischer Mittelalterrezeption zwischen Kanon und Populärkultur. Berlin 2012 (Trends in medieval philology, Bd. 27); Dorothea Klein (Hrsg.), Überall ist Mittelalter. Zur Aktualität einer vergangenen Epoche. Würzburg 2015 (Würzburger Ringvorlesungen, Bd. 11).

25 Z. B. Buck/Brauch (Hrsg.), Mittelalter zwischen Vorstellung und Wirklichkeit (wie Anm. 16); Christian Rohr (Hrsg.), Alles heldenhaft, grausam und schmutzig? Mittelalterrezeption in der Populärkultur. Münster 2011 (Austria: Forschung und Wissenschaft. Geschichte, Bd. 7); im Hinblick auf Präkonzepte Friederike Stöckle, „Die armen kleinen Bäuerlein - was die für 'ne Ausbeutung über sich ergehen lassen mussten....". Alltagsvorstellungen zum Mittelalter: Verständnisbarrieren oder hilfreiche Erklärungsmuster, in: Buck/Brauch (Hrsg.), Mittelalter zwischen Vorstellung und Wirklichkeit (wie Anm. 16), 301-310.

26 Monika Fenn (Hrsg.), Frühes historisches Lernen. Projekte und Perspektiven empirischer Forschung. Frankfurt a. M. 2018 (Geschichtsunterricht erforschen, Bd. 7).

27 Markus Drüding/Tobias Enseleit, Ritter, Hexen, Pest und Burgen? Eine explorative Studie zu Mittelalter-Vorstellungen und dem Mittelalter-Verständnis von Geschichtsstudierenden, in: Zeitschrift für Didaktik der Gesellschaftswissenschaften 8, 2017, 98; Sebastian Barsch/Burghard Burghard, „Es bereitet sie vor, an den richtigen Stellen zu staunen, wenn sie die Tageszeitung lesen" - Historisches Fachwissen aus Perspektive von Mediävist*innen, in: Zeitschrift für Geschichtsdidaktik 18 (2019), 78-96.

28 Vgl. die Übersicht bei Ingrid Paus-Hasebrink, Forschen mit Kindern und Jugendlichen, in: Lothar Mikos/ Claudia Wegener (Hrsg.), Qualitative Medienforschung. Ein Handbuch. 2. völlig überarb. Aufl. Konstanz 2017, 276-282; Markus Kübler betont die Wichtigkeit der Berücksichtigung der sprachlichen Fähigkeiten der Kinder bei Erhebungen, die durch Einzelbefragungen kostenintensiv werden, Markus Kübler, Erhebungsmethoden zum historischen Denken und Lernen bei vier- bis zehnjährigen Kindern, in: Fenn (Hrsg.), Lernen (wie Anm. 26), 43, 50.

29 Monika Pape, Geschichtsbewusstsein im Grundschulalter: eine empirische Studie, in: https://www.widerstreit-sachunterricht. de/ Ausgabe Nr. 11/Oktober 2008, S. 1 Anm. 1 (letzter Zugriff: 26.5.2021); eine Übersicht zur Forschungsgeschichte bietet Bodo von Borries, Streiflichter auf ältere Erkundungen „Frühen historischen Lernens”. Alltagserfahrungen, Forschungszugriffe, Deutungskontroversen, in: Fenn (Hrsg.), Lernen (wie Anm. 26), 21-40. 
nur in Deutschland zu beobachten ist. ${ }^{30}$ Die mediävistische Geschichtswissenschaft hat sich mit diesem Komplex kaum befasst, obwohl das Mittelalter der Ritter und Burgen - meistens gespeist aus Sachbüchern - das Interesse von Grundschüler*innen weckt. ${ }^{31}$ Wenn also die Geschichtswissenschaft Kinder-Mittelalterbilder bisher nicht als einen möglichen Untersuchungsgegenstand betrachtet hat und die Grundschuldidaktik erst langsam die Möglichkeiten diesbezüglich auslotet, wird die Diskrepanz zwischen Mittelalter-Boom und wissenschaftlicher Beschäftigung noch verstärkt. Schließlich setzt die Werbeindustrie mittelalterliche Assoziationen für das Marketing von Kinderprodukten, wie z. B. Ritter-Tütensuppen, ein. ${ }^{32}$ Ein Beispiel für Geschichtskultur, das kaum intensive wissenschaftliche Beratung in Anspruch genommen hat, wirbt also mit Mittelalterbildern, deren Einsatz kommerziell erfolgversprechend sein muss. ${ }^{33}$ Auch Museen bieten zunehmend spezifische Produkte in ihren Shops für unterschiedliche Altersgruppen von Kindern und Jugendlichen an, ${ }^{34}$ sodass sich Lehrende überlegen sollten, ob dieses Prinzip in modifizierter Weise nicht auch für die Lehre nutzbar gemacht werden könnte. Dabei könnten wir unsere Möglichkeiten zu einem professionellen Beitrag zu gesamtgesellschaftlichen Diskursen prüfen. ${ }^{35}$

\section{Überlegungen zum Einsatz in der Hochschullehre}

Ausgehend von diesen Überlegungen sollten in einer universitären Lehrveranstaltung die Möglichkeiten, Chancen und Herausforderungen des Einsatzes von kindlichen Mittelalterbildern der Geschichtskultur erprobt werden. Kinder wurden deshalb gewählt, weil deren Präkonzepte vor dem Schulunterricht ausgeprägt werden und damit einen

30 Markus Kübler, Frühes Historisches Denken bei 4- bis 10-jährigen Kindern in der deutsch-, italienisch- und romanischsprachigen Schweiz - ein Werkstattbericht, 2010, http://www.historischesdenken.ch/assets/files/historisches_denken_ GDSU_2010.pdf (letzter Zugriff: 26.5.2021).

31 Pape, Geschichtsbewusstsein (wie Anm. 29), $4 \mathrm{f}$.

32 Vom Lebensmittelkonzern Nestlé unter der Marke ,Maggi' als, Ritter Suppe mit abenteuerlichen Ritternudeln' seit einigen Jahren vertrieben: Zentral abgebildet ist eine Suppenschale mit Suppe und Löffel. Am linken Rand über die Hälfte der Tütenhöhe ausgestreckt sitzt ein grüner Drache mit lila Kamm und Horn im Profil nach rechts blickend und die Vorderläufe Richtung Teller ausstreckend. Die rosa Zunge ist aus dem geöffneten Maul zur Suppe hinausgestreckt. Der Schwanz rahmt den Suppenteller ein. Auf der rechten Seite oberhalb des Suppentellers reitet ein Ritter auf einem weißen Pferd in voller Plattenrüstung (ca. 16. Jh.) mit rot-weißer Lanze und rotem Löwen auf dem Schild auf den Teller zu. Im Hintergrund ist eine grüne, hügelige Landschaft mit stilisierten Nadelbäumen und einer stilisierten Burg zu erkennen. Die aktuelle Gestaltung der Verpackung verschiebt den Suppenteller auf die linke Hälfte der Verpackung. Auf der rechten Hälfte ist der gleiche Drache spiegelverkehrt und verkleinert abgebildet, der ein Schild mit der bunten Aufschrift „Für Kindergeschmack entwickelt" hält. Die Hügellandschaft mit etwas veränderter Burg wurde stark verkleinert zwischen dem Produktnamen und dem Drachen eingefügt. Der namengebende Ritter fehlt. Die Werbebotschaft zur Freiheit von Geschmacksverstärkern wurde durch „Einfach gut mit abenteuerlichen Ritternudeln eigener Herstellung" ersetzt.

33 Werbung folgt der AIDA-Formel: Attention, Interest, Desire, Action (=Kaufhandlung). Vgl. Christine Feil, Kinder, Geld und Konsum. Die Kommerzialisierung der Kindheit. Weinheim/München 2003 (Kindheiten, Bd. 24).

34 Peter Leimgruber/Hartmut John, Museumsshop-Management. Einnahmen, Marketing und kulturelle Vermittlung wirkungsvoll steuern. Ein Praxis-Guide. Bielefeld 2011, 232f. Das ist kein spezifisch deutsches Phänomen: Bspw. bot der Museumsshop der Ruine von Kirkstall Abbey bei Leeds in Großbritannien im Juli 2019 u. a. Produkte der Firma Pillow Fight Warrior an; https://www.pillowfightwarriors.com/ (letzter Zugriff: 26.5.2021).

35 Eine solche Beteiligung als ,citizens' fordern Jones/Kostick/Oschema, Why Should we Care (wie Anm. 5), 14f. ,Wir' meint die Gemeinschaft der Hochschullehrenden mit den Hochschullernenden, die teilweise als Lehrkräfte an Schulen gehen werden. 
Ausgangspunkt für den reflektierten Umgang mit dem Mittelalter in der Schule bilden können. ${ }^{36}$ Dabei sollte auch geprüft werden, ob die Diskussion dieser Präkonzepte in der Hochschullehre geeignet erscheint, das Reflexionsniveau zu Geschichtsbildern zu steigern und damit die Bereitschaft zur Änderung von eigenen Präkonzepten zu erhöhen. Es besteht nämlich der Eindruck, dass sowohl Schüler*innen als auch Studierende stark von Präkonzepten geprägt sind. Die empirische Datenlage ist gegenüber der gefühlten Wahrnehmung jedoch dünn. ${ }^{37}$ Einzelne Erhebungen zu den Mittelalterbildern von Studierenden der Geschichtswissenschaft, Schüler*innen und Erwachsenen haben kaum relevante Unterschiede festgestellt. ${ }^{38}$ Einerseits ist die Beschäftigung mit dieser Thematik für Lehramtsstudierende, die die Mehrzahl der Studierenden der Geschichtswissenschaft an den meisten deutschen Hochschulen ausmachen, ${ }^{39}$ von besonderem Interesse, weil sie so die Möglichkeit erhalten, sich auf Mittelalterbilder ihrer zukünftigen Schüler*innen und deren Chancen und Herausforderungen des Einsatzes im Unterricht vorzubereiten. Andererseits lohnt sich für angehende Historiker*innen mit Berufszielen außerhalb der Universität eine Beschäftigung mit der Beziehung von verschiedenen Typen von Mittelalterbildern, weil beispielsweise Museen und Medien verschiedene Rezeptionsansprüche und -fähigkeiten bedienen müssen. Dies kann in Veranstaltungen zur Rezeptionsgeschichte geübt werden, in denen Lehrende und Studierende allgemeine Mittelalterbilder mit Ansätzen des Forschenden Lernens untersuchen. ${ }^{40}$

Empirische Daten der Geschichtsdidaktik haben in verschiedenen Studien für Kinder in Grundschulen und der frühen Sekundarstufe I ein hohes Interesse an mittelalterliche Themen festgestellt, die mit Attributen wie ,fern', ,abenteuerlich` oder ,anders“ beschrieben werden. ${ }^{41}$ Vor allem für angehende Lehrkräfte erscheint also die Beschäftigung mit Kinder-Mittelalterbildern lohnenswert, da diese ihre performative Kraft bereits entfaltet haben, bevor die Schüler*innen Mittelalterunterricht erhalten. Also könnte es

36 Buck, Mittelalter (wie Anm. 12), 183.

37 Paul Sturtevant hat in einer Studie Zusammenhänge zwischen Mittelalterbildern aus Disneyfilmen und denjenigen von jungen Erwachsenen in Großbritannien aufgezeigt. Vgl. Paul Sturtevant, „You don't learn it deliberately, but you just know it from what you've seen". British understandings of the medieval past gleaned from Disney's Fairy tales, in: Tison Pugh/Susan Aronstein (Hrsg.), The Disney Middle Ages. A Fairy-Tale and Fantasy Past. New York 2012 (The New Middle Ages), 77-96, vor allem 78, $92 \mathrm{f}$.

38 Drüding/Enseleit, Ritter (wie Anm. 27), 114.

39 An der CAU durchschnittlich zwei Drittel der Geschichtsstudierenden.

40 Vgl. Andreas Bihrer/Stephan Bruhn/Fiona Fritz, Forschendes Lernen in Geschichtswissenschaft und Geschichtsstudium. Kompetenzen - Rahmenbedingungen - Projekte - Perspektiven, in: Margrit E. Kaufmann/Ayla Satilmis/Harald A. Mieg (Hrsg.), Forschendes Lernen in den Geschichtswissenschaften. Konzepte, Praktiken und Perspektiven hermeneutischer Fächer. Wiesbaden 2018, 105-124.

41 Helmut Beilner: Erkundungen zum Geschichtsbewusstsein am Ende der Grundschulzeit, in: Waltraut Schreiber (Hrsg.), Erste Begegnungen mit Geschichte. Grundlagen Historischen Lernens 1. Neuried 1999, 133; Thoralf Schenk: Der Zug ist abgefahren (...). Konzeption und Zwischenergebnisse einer Untersuchung zu geschichtlichen Interessen und historischen Vorstellungen von Schülern im Primar- und Sekundarstufenbereich, in: Zeitschrift für Geschichtsdidaktik 6, 2007, 177; Sebastian Barsch, Luxus in Pink? Playmobilprinzessinnen, Geschlechtervorstellungen und Geschichtsbewusstsein. In: Christoph Kühberger (Hrsg.): Mit Geschichte spielen. Zur materiellen Kultur von Spielzeug und Spielen als Darstellung der Vergangenheit. Bielefeld 2021 (im Druck). 
für die weitere schulische Entwicklung des Interesses an Geschichte im Allgemeinen und am Mittelalter im Speziellen von Bedeutung sein, die Erwartungen der Schüler*innen in der Unterrichtsgestaltung zu berücksichtigen und nutzbar zu machen. ${ }^{42}$

Außerdem sollte in der Veranstaltung geklärt werden, welche Mittelalterbilder Studierende in das Studium aus der Schule mitbringen und ob Studierende Zusammenhänge mit außer- oder vorschulischen Berührungen mit der mittelalterlichen Geschichte sehen, die mutmaßlich der Geschichtskultur zuzuordnen sind. Ein Ziel war die Diskussion von Vorschlägen, wie klischeebehafteten Mittelalterbildern ${ }^{43}$ sinnvoll und nachvollziehbar begegnet werden könne, um geschichtswissenschaftliche Mittelalterbilder in die Gesellschaft zu tragen.

Für die Hochschullehre besteht darin eine Chance, den Zweck von Theorie und Praxis in gezielten Anwendungsbeispielen deutlich zu machen und damit das Interesse an der Veranstaltung zu erhöhen. Schließlich zählt die Thematisierung und Diskussion von unterschiedlichen Darstellungsformen von Geschichte - Geschichtskulturen sind eine Darstellungsform von Geschichte - zu den zentralen Aufgaben eines Geschichtsstudiums. Durch die Beschäftigung mit diesen wird der Blick für Übereinstimmungen und Unterschiede sowie Wechselwirkungen geschärft, sodass die Kommunikation zwischen Geschichtswissenschaft und Gesellschaft stärker davon als von OpenAccess profitieren könnte. ${ }^{44}$

Im Wintersemester 2018/19 bot ich eine Übung im Methodik-Modul mit dem Titel ,Ritter und Prinzessin? Mittelalterbilder von und für Kinder` an. Im Verlaufsplan des B.A. ist diese Veranstaltung zwischen dem vierten und sechsten Semester vorgesehen und nicht epochal gebunden. Daher war davon auszugehen, dass der Anteil von intrinsisch motivierten Studierenden mit grundsätzlichem Interesse am Mittelalter höher als im durchschnittlichen Proseminar sein würde, das alle Studierenden absolvieren müssen. Der Titel stand für das zu erwartende Kondensat von Mittelalterbildern. Jedoch wäre der Titel ,Ritter und Burgen` treffender gewesen. Eine Stichprobe in der Bücherei der Stadt Elmshorn ${ }^{45}$ ergab für das Regalbrett in der Rubrik ,Sachbuch, Geschichte, Mittelalter', dass 18 von 27 Büchern ,Burg und/ oder Ritter' im Titel führten. Von den neun verbliebenen hatten noch fünf weitere Ritter und/ oder eine Burg auf dem Cover.

42 Buck, Mittelalter (wie Anm. 12), 81f.; Schenk warnt, dass durch eine frühe Dekonstruktion von Vorstellungen bei Schüler*innen deren Interesse an der Vergangenheit schwinde; Schenk, Der Zug ist abgefahren (wie Anm. 41), 177; Stöckle spricht sich für die Nutzung der aus der Alltagswelt der SuS stammenden Vorstellungen und Konzepte aus, Stöckle, „Die armen kleinen Bäuerlein" (wie Anm. 25), 310.

43 Vor allem die Vorstellung, dass im Mittelalter alle Menschen dachten, dass die Welt eine Scheibe wäre, ist aus Sachbüchern kaum wegzudenken: Andrea Schaller, Mittelalter. Die Welt der Kaiser, Edelleute und Bauern. Nürnberg 2013 (WAS IST WAS, Bd. 118), 7; Jutta von der Lühe, Diktate 3. Klasse. Deutsch. Lerne mit Dinosauriern, Detektiven und mehr. Stuttgart 2015, 27; Roland Bernhard: Der Eingang des „Mythos der flachen Welt" in deutsche und österreichische Geschichtsschulbücher im 20. Jahrhundert, in: GWU 63 (2013), 687-701.

44 Das Bundesministerium für Bildung und Forschung warb im Februar 2020 damit, dass Open Access Elfenbeintürme abbaue, https://www.bildung-forschung.digital/de/open-access-initiativen-2680.html (letzter Zugriff: 26.5.2021); auch Jones/ Kostick/Oschema, Why Should we Care (wie Anm. 5), 21, fordern mehr Engagement seitens der Geschichtswissenschaft, um die Öffentlichkeit an ihren internen Debatten teilhaben zu lassen.

45 Durchgeführt am 15.11.2019 und mit ähnlichen Ergebnissen im Dezember 2019 und Januar 2020 wiederholt. Die schleswigholsteinische Stadt hat ca. 50000 Einwohnende und liegt in der Metropolregion Hamburg. 
Auch Untersuchungen von Nicola Brauch zu vorunterrichtlichen Wahrnehmungen bei Sechstklässler*innen bestätigen diesen Befund, ergänzt um Personen, Ereignisse und Räume aus dem Bereich, der im weitesten Sinne mit höfischer Kultur zu umschreiben ist: Pferde, Turniere, Feste, Hofdamen, Könige und Fürsten sowie Schlachten und Waffen, Rüstungen und Kerker. ${ }^{46}$ Diese Aspekte unterscheiden sich kaum von Mittelalterbildern der Allgemeinbevölkerung, die dem positiven, romantisierenden Bild einer vermeintlich urtümlich-exotischen Zeit jenseits von Unwägbarkeiten moderner Gesellschaftsentwicklungen zuzuordnen sind. ${ }^{47}$ Der Kerker steuert dann einen Hauch schauriges, negatives Mittelalter bei. ${ }^{48} \mathrm{Zu}$ ähnlichen Ergebnissen führen Mittelalter-Brainstormings in Proseminaren. Unter der Überschrift ,Dunkle Zeit` - zugegebenermaßen eine suggestive Beeinflussung, die jedoch bewirken soll, dass Assoziationen ohne Vorüberlegung im Hinblick auf die mögliche Bewertung der Dozentin genannt werden - zählen Studierende folgende Aspekte auf: Ritter, Burgen, Stadt, Kirche, Ständegesellschaft und ihre Vertreter, Pest und Krieg - vor allem am Beispiel der Kreuzzüge. In Bezug auf Kirche und Hygiene ist dabei eine skeptische bis negative Haltung, also ein negatives Mittelalterbild, erkennbar. Markante Abweichungen unter den genannten Aspekten erklären sich fast immer mit dem Schulort der Studierenden. ${ }^{49}$ Grundsätzlich sind mit Stadt, Ständegesellschaft und Kreuzzügen die Mittelalter-Themen der Fachanforderungen bekannt. ${ }^{50}$

\section{Ziele und Voraussetzungen für die Konzeption und Durchführung der Übung}

Inhaltlich diente die Übung zwei Zielen: Einerseits galt es festzustellen, ob es überhaupt andere Mittelalterbilder neben ,Ritter und Burgen' gibt. Andererseits sollte geprüft werden, ob Variationen oder Unterschiede je nach Medium und Alter der Kinder festgestellt werden können und wie diese für die Reflektion von Geschichtsbewusstsein nutzbar gemacht werden können.

\footnotetext{
46 Brauch, Die vergessenen Fragen (wie Anm. 11), 177f.; Pape hat bei Grundschüler*innen die gleichen Aspekte festgestellt: Pape, Geschichtsbewusstsein (wie Anm. 29), 4.

47 Vgl. wissenschaftstheoretisch Otto-Gerhard Oexle, Die Gegenwart des Mittelalters. Berlin 2013 (Das Mittelalterliche Jahrtausend, Bd. 1), 6-19; rezeptionsgeschichtlich orientierte Typenbildung bei Umberto Eco, Faith in Fakes. Travels in Hyperreality. London 1998 (Nachdruck von 1983), 68-72; auf Basis von Umfragen bei Schüler*innen in den 90er Jahren Bodo von Borries, Das Mittelalter im Geschichtsbewusstsein von Jugendlichen, in: Ballof (Hrsg.) (wie Anm. 16), 280-283.

48 Das wird mitunter bewusst für Veröffentlichungen genutzt: Fiona Macdonald/David Antram, Zum Glück lebst Du nicht im Mittelalter! Begegnungen, auf die du gut verzichten kannst. Hamburg 2007.

49 Beispielsweise wird von Studierenden, die in Lübeck zur Schule gegangen sind, gelegentlich der Knochenhauer-Aufstand von 1384 erwähnt.

50 Die Orte der Schulabschlüsse wurden nicht abgefragt. Doch wie in Ministerium für Bildung, Wissenschaft und Kultur des Landes Schleswig-Holstein (Hrsg.), Leitfaden zu den Fachanforderungen Geschichte. Allgemein bildende Schulen, Sekundarstufe I, Sekundarstufe II. Kiel 2018, 16; 24f., 27, 29f., und Freie und Hansestadt Hamburg Behörde für Schule und Berufsbildung (Hrsg.), Bildungsplan Gymnasium Sekundarstufe I. Geschichte. Hamburg 2011, 26, sowie Freie und Hansestadt Hamburg Behörde für Schule und Berufsbildung (Hrsg.), Bildungsplan gymnasiale Oberstufe. Geschichte. Hamburg 2011, 14, sind die genannten Aspekte in den meisten deutschen Fachanforderungen zu finden.
} 
Zu den methodischen Zielen gehörte die Wiederholung und Vertiefung der Fähigkeiten von Lese- und Schreibmethoden zur dauerhaften Aufbereitung von Wissen für eine weitere Verarbeitung im Hinblick auf Abschlussarbeiten. Eine wichtige Voraussetzung war dabei die Bereitschaft zur Einarbeitung in bekannte und unbekannte Themenkomplexe des eigenen Faches und anderer Fächer. Dadurch sollte auch Wissen in mittelalterlichen Bereichen generiert oder vertieft werden.

Als Richtwert für die Definition von Kindheit nutzten wir im Kurs Artikel 1 des Deutschen Jugendschutzgesetzes, das Personen, die noch nicht 14 Jahre alt sind, als Kinder bezeichnet. Nach theoretischen Vorüberlegungen zu Geschichtstheorie und Mittelalterbildern untersuchten wir verschiedene Medien nach gattungstheoretisch sortierten Blöcken - und zwar von vermeintlicher Fachnähe hin zu steigender Kommerzialisierung:

Im ersten Block ,Literatur` widmeten wir je eine Sitzung Bilder-, fiktionalen und Sachbüchern, wobei eine eindeutige Unterscheidung schwierig war. Interdisziplinär betrachtet bestand dabei die größte Überschneidung zu den Kompetenzen der Studierenden, weil die Nähe zwischen Literatur- und Geschichtswissenschaft als hoch anzusehen ist. Außerdem hatten zwei Drittel der Studierenden ein literaturwissenschaftliches Zweitfach.

Der zweite Block ,Spiele und Museen' thematisierte das lebendige Mittelalter anhand von Spielen, Spielzeug und Mittelalter-Märkten sowie ein wachsendes Segment in Museen, das sich in eigenen Programmen, Ausstellungen und Katalogen für Kinder zeigt. ${ }^{51}$ Mit Museen gehörte zwar ein Berufsfeld der Geschichtswissenschaft zu diesem Block, doch nehmen Museologie und Museumspädagogik nicht in jeder Veranstaltung im Studium zentralen Raum ein. ${ }^{52}$ Wichtige Berührungspunkte können Ansätze der Materialität liefern, die auch Möglichkeiten für den Einsatz der Historischen Grundwissenschaften bieten, oder kulturanthropologische Untersuchungen..$^{53}$ Jedoch müssen die Hersteller*innen von Spielen und Spielzeugen wie Verlage ökonomische Belange berücksichtigen.

Den letzten Themenkomplex bildeten Produkte aus Film, Fernsehen und Multimedia, die explizit für Kinder produziert worden sind. Auch wenn es sich um eine Form der Darstellung von mittelalterlicher Geschichte handelt, spielen medientheoretische

\footnotetext{
51 Das Europäische Hansemuseum in Lübeck bot für Kindern ab sechs Jahren vom 2.12.2018 bis zum 3.3.2019 die Sonderausausstellung, Segel, Salz und Silberlinge. Seehandel in der Hansezeit' an. Dort sind auch Programme für Kinder-Geburtstage zu buchen: Abenteuer Hansefahrt - Gemeinsam sind wir stark!, https://www.hansemuseum.eu/wp-content/uploads/2020/05/ Infoblatt-Kindergeburtstage.pdf (letzter Zugriff: 26.5.2021); ohne Angebote für Kinder kommen die größeren Museen nicht mehr aus, z. B. am 5.1.2020 im Germanischen Nationalmuseum Nürnberg laut Programmheft: ,Kleine Entdecker im Museum'.

52 Der Praxis-Anteil ist an den Historischen Seminaren an deutschen Universitäten individuell geregelt. Die am Historischen Seminar der Christian-Albrechts-Universität zu Kiel angebotenen Projektseminare können diesen Bereich konkretisieren.

53 Ein Beispiel für die interdisziplinäre Zusammenarbeit ist die Tagung ,Mit Geschichte spielen. Zur materiellen Kultur von Spielzeug und Spielen als Darstellung der Vergangenheit', veranstaltet durch das Salzburg Museum, das dortige Spielzeug Museum und die Universität Salzburg vom 13.11.2019 bis zum 15.11.2019 in Salzburg, vgl. den Tagungsbericht von Antonia Grage/ Tomke Jordan, Mit Geschichte spielen. Zur materiellen Kultur von Spielzeug und Spielen als Darstellung der Vergangenheit, 13.11.2019-15.11.2019 Salzburg, in: H-Soz-Kult, 8.1.2020, www.hsozkult.de/conferencereport/id/tagungsberichte-8581 (letzter Zugriff: 25.05.2021).
} 
Erwägungen ${ }^{54}$ eine größere Rolle als in der universitären Mediävistik. Auch die Vermarktungsstrategien im Hinblick auf den kommerziellen Erfolg haben eine wichtige Bedeutung, weil hierfür eher das Bekannte und Vertraute als der aktuelle Forschungsstand genutzt zu werden scheint. ${ }^{55}$

Hochschuldidaktisch bot diese Veranstaltung also die Möglichkeit, die allseits geforderte Interdisziplinarität mit Studierenden anhand von Beispielen zu erproben und damit Chancen und Herausforderung zu identifizieren. Gleichzeitig konnten die Kenntnisse und Fähigkeiten theoretischer Prämissen, inhaltlichen Wissens oder methodischer Ansätze im eigenen Fach angewendet und geprüft werden.

\section{Ergebnisse und Folgerungen aus der Kategorie Sachbuch}

\section{Ritter und Burgen}

Sachliteratur ${ }^{56}$ soll im Folgenden vor allem als Beispiel dienen, weil der Zweck der Information den mutmaßlich höchsten Grad an Überschneidung mit geschichtswissenschaftlicher Arbeit hat. Als wiederkehrende Themenschwerpunkte von Mittelalterbildern sind Ritter und Burgen, Adel und Bauern, die Wikinger sowie die Pest zu nennen. Ob die Hanse nur in Norddeutschland ein unter Kindern bekanntes Phänomen ist, war bisher nicht abschließend zu klären. Wie erwartet haben Ritter und Burgen den größten Bekanntheitsgrad. Daher werden diese Beispiele in den Mittelpunkt gestellt.

Als erste Schlussfolgerung und Einschränkung ist festzuhalten, dass das Mittelalterbild von Kindern innerhalb solcher Veranstaltungen nur schwer zu ermitteln ist. Einzelbefragungen von eigenen Kindern, Nichten oder Neffen sowie Patenkindern etc. können zwar Hinweise und Ergänzungen bieten. Allerdings haben wir die Erfahrung ${ }^{57}$ gemacht, dass anwesende Erwachsene (oft Eltern oder Großeltern) recht schnell in die Verbalisierungsversuche der Kinder eingreifen. Wenn das Kind dann nur noch zustimmt, ist kaum zu ermitteln, ob das Mittelalterbild des Kindes von der Verbalisierung

54 Edgar Lersch/Reinhold Viehoff, Geschichte im Fernsehen. Eine Untersuchung zur Entwicklung des Genres und der Gattungsästhetik geschichtlicher Darstellungen im Fernsehen 1995 bis 2003. Berlin 2007 (Schriftenreihe Medienforschung der Landesanstalt für Medien Nordrhein-Westfalen, Bd. 54); mit Schwerpunkt in Zeitgeschichte und Dokumentarfilm als Quelle: Carsten Heinze/Arthur Schlegelmilch (Hrsg.), Der dokumentarische Film und die Wissenschaft. Interdisziplinäre Betrachtungen und Ansätze. Wiesbaden 2019 (Filme und Bewegtbild in Kultur und Gesellschaft).

55 In Bezug auf die Ästhetik von Bilderbüchern des 20. Jahrhunderts vgl. Jens Thiele, Das Bilderbuch. Ästhetik - Theorie Analyse - Didaktik - Rezeption. Oldenburg 2000, 16. Das ist auch auf andere Produkte übertragbar.

56 Das Kinder-Sachbuch soll informieren und nutzt dabei Aspekte der Fiktionalität und der Faktualität in belehrender und unterhaltender Form. Vgl. Sabine Berthold, Creating History. Zeitreisen ins Mittelalter in der Sachliteratur für Kinder und Jugendliche, in: Ingrid Bennewitz/Andrea Schindler (Hrsg.), Mittelalter im Kinder- und Jugendbuch. Akten der Tagung Bamberg 2010. Bamberg 2012 (Bamberger interdisziplinäre Mittelalter-Studien, Bd. 5), 116.

57 Es handelt sich nicht um quantitativ gesicherte Ergebnisse, sondern Eindrücke der Studierenden und Lehrperson. 
der Erwachsenen abweicht. ${ }^{58}$ Außerdem reicht die Zeit einer einsemestrigen Lehrveranstaltung nicht aus, um eine valide empirische Untersuchung zu leisten. Zudem entstehen die Mittelalterbilder von Kindern nicht voraussetzungslos, sondern sind geprägt durch eben jene Bezugspersonen: Inwieweit Konsum etwa von Werbung ermöglicht oder die Beschäftigung mit dem Mittelalter angeregt wird, hängt maßgeblich von Erziehungsberechtigten und anderen Bezugspersonen einschließlich Erzieher*innen und Lehrpersonen, die als „Informationsvermittler“59 agieren, ab. Es sind also innerhalb einer solchen Veranstaltung eher die Mittelalterbilder zu eruieren, die Kindern von Erwachsenen vermittelt werden ${ }^{60}$ und weniger diejenigen, die Kinder im Spiel daraus machen.

Außerdem ist bei der Analyse zu berücksichtigen, dass sowohl der Zweck als auch die Intention der Sachbücher nicht allein in historischer Bildung besteht: Oft ist diese eher zweitrangig, weil Kinder zählen lernen oder motorische Fähigkeiten und räumliches Vorstellungsvermögen trainieren sollen. ${ }^{61}$ Bei Diktat-Lern-Heften, ${ }^{62}$ die thematisch aufgebaut sind, kann historische Bildung ein Nebenprodukt sein, wenn spannende und lehrreiche Geschichten den primären Zweck der Schreibübungen unterstützen sollen. Spannung und Abenteuer am mittelalterlichen Beispiel dienen in erzählenden Texten auch der gesellschaftlich-sozialen Erziehung und Gruppensozialisation. Dann dient das Mittelalter weniger als Interessensschwerpunkt, sondern eher als austauschbare Kulisse. ${ }^{63}$ Ähnliches gilt für Kinderromane, wie etwa Abenteuer-, Zeitreise- und Kriminalgeschichten. ${ }^{64}$ Allerdings kann die Kulisse ,Mittelalter ${ }^{c}$ hier Wissen über Personen, Orte und bekanntere Themen vermitteln, die in der Grundschule kaum und in der Sekundarstufe I aufgrund der Kompetenzorientierung keine explizite Rolle spielen müssen.

Eine zweite wichtige - wenn auch nicht überraschende - Feststellung ist, dass der geschichtswissenschaftliche Einfluss auf sämtliche untersuchte Medien äußerst gering ist. Ausnahmen bilden hier die Museumskataloge, so wurde z. B. der Katalog

58 Kinder- und Jugendliteratur kann auch als Medium erwachsener Erinnerungskultur gelesen werden. Vgl. Hans-Heino Ewers, In die eigene Kindheit zurücksinken. Kinder- und Jugendliteratur als Medium der (erwachsenen) Erinnerungskultur, in: Gabriele von Glasenapp/Gisela Wilkending (Hrsg.), Geschichte und Geschichten. Die Kinder- und Jugendliteratur und das kulturelle und politische Gedächtnis. Frankfurt a. M. 2005 ((Kinder- und Jugendkultur, -literatur und -medien: Theorie - Geschichte - Didaktik, Bd. 41), 129-142.

59 Andrea Becher/Eva Gläser: Präkonzepte von Grundschulkindern zur historischen Methodenkompetenz. Zentrale Ergebnisse des Forschungsprojekts "HisDeKo", in: Fenn, Lernen (wie Anm. 26), 84.

60 Entgegen den geschichtskulturellen Angeboten mit klaren Schwerpunkten in der Vormoderne stellt Stefanie Zabold eine starke Präsenz der Zeit des Nationalsozialismus im historischen Denken von Kindern fest, die Lehrkräfte nicht erwarten. Stefanie Zabold, Ausprägungen historischen Denkens vor dem ersten Geschichtsunterricht, in: Fenn, Lernen (wie Anm. 26), 61f.

61 Hazel Maskell (Text)/Teri Gower (Illustration und Gestaltung)/Heike Osborne (dt. Übersetzung): Mein Großer Such- und Zählspaß. Ritter und Burgen. London 2013; Alice Pearcey: Ritter und Burgen Puzzlebuch, hg. v. Anna Milbourne, Wien 2007; beide beraten von Abigail Wheatley, The Idea of the Castle in Medieval England. York 2004.

62 Theo Kaufmann, Diktate 4. Klasse. Deutsch. Lerne mit Piraten, Drachen und mehr. Stuttgart 2015, 26-29, beworben auf dem Cover mit "Coole Themen für bessere Noten", wozu auch Ritter gehören.

63 So beispielsweise von Cornelia Funke, Igraine Ohnefurcht', vgl. Ina Karg, Ritter, Elfen, Zauberwelten. Mittelalterbilder in aktuellen Kinder- und Jugendbüchern, in: Volker Mertens/Carmen Stange (Hrsg.), Bilder vom Mittelalter. Eine Berliner Ringvorlesung. Göttingen 2007 (Aventiuren Sonderband), 164.

64 Vgl. v. Fabian Lenk die Reihe ,Zeitdetektive', erschienen in Ravensburg: Das Teufelskraut 4, Der Schatz der Wikinger 7, Das Silber der Kreuzritter 9, Marco Polo und der Geheimbund 11, Freiheit für Richard Löwenherz 13 (alle 2013 als E-Book), Gefahr am Ulmer Münster 19 (5. Aufl. 2010), Der Fluch der Wikinger 24, Der Betrüger von Lübeck 26, Entführung in Nürnberg 29 (alle 2014 als E-Book), Barbarossa und der Raub von Köln 34 (2016 als E-Book), Der letzte Ritter von Füssen 41 (2019). 
,Die Burgen-Ratten sind los!' zur ,Mythos Burg'-Ausstellung in Nürnberg (2010 in Kooperation mit dem Deutschen Historischen Museum in Berlin ,Burg und Herrschaft') von Anja Grebe maßgeblich betreut, die inzwischen Professorin für Kulturgeschichte und Museale Sammlungswissenschaften an der Donau-Universität Krems ist.

Der bekannte französische Mediävist Jacques LeGoff erklärt in ,Das Mittelalter für Kinder ${ }^{c}$ die wunderbare Welt der Zeit. ${ }^{65}$ Mit der Altersempfehlung ab 12 Jahren ${ }^{66}$ gehört das Buch aber nur noch knapp zur Kindersachliteratur und wird auch von Kundenrezensent*innen als ungeeignet für Kinder bewertet. ${ }^{67}$ Mit der eher altmodischen Art, auf eine Frage eine erzählende Antwort zu geben, entspricht das Buch vielleicht nicht mehr den Ansprüchen einer durch Multimedia geprägten Kindergeneration. Ansonsten kommen die Verfasser*innen von Bilder- und Sachbüchern sowie Kinderromanen oft aus den Bereichen Pädagogik, Journalismus oder Publizistik und sind teilweise mit einem Studium der Geschichte, Kunstgeschichte, Germanistik oder Psychologie ausgestattet ${ }^{68}$ :

Die erste Ausgabe des Burgen-Bands ${ }^{69}$ der bekannten WAS IST WAS-Reihe ist von Hans-Peter von Peschke, einem Historiker und Journalisten, der zur Firma Siemens promovierte. ${ }^{70}$ Die zweite Ausgabe hat die Kunsthistorikerin und freie Autorin Andrea Schaller, die zur Ikonographie des Erzengels Michael im frühen Mittelalter promovierte, ${ }^{71}$ verfasst. Bezeichnenderweise gibt der Tesloff-Verlag inzwischen - bei der ersten Auflage wird der Autor als Historiker auf dem Buchrücken geführt - kaum noch Hinweise auf das Studium oder Ausbildung der jeweiligen Autor*innen auf dem Buchrücken oder auf der Homepage an. ${ }^{72}$

Wissenschaftlichkeit oder Expertise der Verfassenden scheint also kein wichtiges Verkaufskriterium für Kinder-Sachbücher in Deutschland zu sein. Allerdings tritt in der Neuauflage die Materialität durch Photographien, die viele Zeichnungen ersetzen, deutlicher hervor: ${ }^{73}$ Dabei handelt es sich um die Reihe, die Kinder ab acht Jahren

65 Jacques LeGoff, Das Mittelalter für Kinder, 2. Aufl. München 2007.

66 Auf dem Buchrücken steht noch „ab 10 Jahre"; der Verlag empfiehlt das Buch inzwischen ab 12 Jahre: https://www.chbeck. de/le-goff-mittelalter-kinder/product/21329 (letzter Zugriff: 26.5.2021).

67 Zwei Kundenrezensionen bei Amazon (Dt.) vom 10.7.2011 und 29.11.2011 kritisieren vor allem die fehlende Anschaulichkeit.

68 Hans-Günther Döring beantwortete freundlicherweise meine Frage nach seinen Vorlagen und seiner Inspiration mit einem Hinweis auf zahlreiche meist - aber nicht ausschließlich - populärwissenschaftliche Sachbücher.

69 Hans-Peter von Peschke, Burgen, Nürnberg 1989/2008 ((WAS IST WAS, Bd. 106); zur objektivierenden Vorgehensweise im Band vgl. Berthold, Creating History (wie Anm. 56), $119 \mathrm{f}$.

70 Ders., Elektroindustrie und Staatsverwaltung am Beispiel der Firma Siemens 1847-1914. Frankfurt a. M. (Europäische Hochschulschriften Reihe 3, Bd. 154) 1981.

71 Andrea Schaller, Burgen. Zeugen des Mittelalters. Nürnberg 2014 (WAS IST WAS, Bd. 106); Dies., Der Erzengel Michael im frühen Mittelalter. Ikonographie und Verehrung eines Heiligen ohne Vita. Bern/Berlin/Brüssel 2006.

72 https://shop.tessloff.com/WAS-IST-WAS/WAS-IST-WAS/Buchreihe-NEU/WAS-IST-WAS-Band-106-Burgen.html (letzter Zugriff: 26.5.2021); dagegen der Band 118 Mittelalter. Die Welt der Kaiser, Edelleute und Bauern ist von Dr. (!) Andrea Schaller, https://shop.tessloff.com/WAS-IST-WAS/WAS-IST-WAS/Buchreihe-NEU/WAS-IST-WAS-Band-118-Mittelalter.html (letzter Zugriff: 26.5.2021).

73 In Peschkes Band wird der Illustrator Nikolai Smirnov auf dem Deckblatt genannt. Photographien von der Wartburg, der Wasserburg Chillon und der Kaiserburg in Nürnberg sind nur auf S. 8-9 zu finden. Teilweise sind mittelalterliche Illustrationen genutzt worden. 
anspricht. Sie erscheint bereits seit 1963 als Hardcoverausgabe - zuvor als Monatszeitschrift im Zeitschriftenhandel - und ist laut Selbstaussage des Verlags die erste Kinderund Jugendbuchreihe in Deutschland. ${ }^{74}$ Der Aufbau ist orientiert an moderneren, digitalen Layouts und folgt nicht mehr der bekannten Kolumnenstruktur. Diese Umgestaltung und Neukonzeption begann 2013. Der Verlag reagierte damit auf die veränderten Lese- und Sehgewohnheiten von Kindern. ${ }^{75}$ Es wäre interessant, ob dies Einfluss auf deren kognitive und neuronale Aufnahme und Verarbeitung hat oder ob diese Modernisierung Ursache oder Folge von Veränderungen solcher Prozesse ist. Welchen Einfluss dabei digitale Medien auf narrative Kompetenzen von Schüler*innen haben, ist ein noch junges Forschungsfeld. ${ }^{76}$

Der kleinteiligen Segmentierung der Kindheit, ${ }^{77}$ die die Werbebranche mit dem Ziel der Erhöhung des Konsums vorgibt, trägt auch Tessloff mit den Reihen ,Kindergarten (ab drei Jahren), ,Junior‘ (vier bis sieben Jahren) und ,Erstes Lesen` Rechnung. Bis auf die Reihe ,Erstes Lesen', gibt es in allen Reihen einen Band ,Ritterburg', die ausschließlich mit Zeichnungen illustriert worden sind.$^{78}$ Dass die Bezeichnung ,Ritterburg' unzutreffend ist, ${ }^{79}$ wird erst in der Reihe für die ältesten Kinder erläutert, in der der Band diesen Titel auch nicht führt. ${ }^{80}$ Beide Bände deuten immerhin historische Veränderungen beim Burgenbau an: Die ersten Burgen seien aus Holz und leicht brennbar gewesen, weshalb Steinburgen gebaut worden seien. ${ }^{81}$ Schaller bietet in je einem eigenen Kapitel Baugeschichte und verschiedene Typen von Burgen sowie die Weiterentwicklung in der Neuzeit. ${ }^{82}$ Waffen und Kampf scheinen nicht dem Militarismusverdacht zu unterliegen, denn in beiden Reihen wird die Verteidigungsfunktion von Burgen versachlicht am Beispiel des Burgenaufbaus (z. B. Wehrturm oder Schießscharte) auf den ersten beiden Seiten deutlich. ${ }^{83}$ Weitere behandelte Aspekte sind das Leben und Arbeiten auf der Burg inklusive Feste, Ausbildung und Rüstung eines Ritters, Turniere und schließlich

74 https://www.tessloff.com/was-ist-was-583.html (letzter Zugriff: 26.5.2021); Ragnar Tessloff stieß in den USA auf die Kinder- und Jugendsachbuchreihe ,How and why?', für die er 1959 Lizenzverträge abschloss: https://www.tessloff.com/verlag-582.html (letzter Zugriff: 26.5.2021).

75 Ebd.

76 Dissertationsprojekt von Henning Host: Eine qualitative Analyse des Zusammenhangs zwischen digitalen Medien und der Entwicklung narrativer Kompetenzen von Schülerinnen und Schülern im gymnasialen Geschichtsunterricht bei Sandra Hofhues (FernUniversität Hagen) und Sebastian Barsch (CAU).

77 Vgl. die Übersicht bei Feil, Kinder (wie Anm. 33). 83.

78 Hans-Günther Döring, Ritterburg. Nürnberg 2016 (WAS IST WAS - Kindergarten, Bd. 3); Martin Stiefenhofer (Text)/Barbara Jelenkovich (Illustrationen), Ritterburg, Nürnberg 2017 (WAS IST WAS - Junior, Bd. 6).

79 Thomas Wozniak kritisiert diesen Titel zutreffend auch bei in der Beck'schen Reihe Wissen von Joachim Zeune, Ritterburgen. Bauwerk, Herrschaft, Kultur. München 2015; Thomas Wozniak, Rezension zu: Zeune, Joachim: Ritterburgen. Bauwerk, Herrschaft, Kultur. München 2015. ISBN 978-3-406-66091-7, in: H-Soz-Kult, 10.2.2016, www.hsozkult.de/publicationreview/ $\mathrm{id} / \mathrm{reb}-23660$ (letzter Zugriff 25.5.2021).

80 Schaller, Burgen (wie Anm. 71), 6: „Unglaublich: Das Wort «Ritterburg” gab es im Mittelalter noch nicht. Meist gehörten Burgen dem König oder Adeligen, der jedoch nicht unbedingt Ritter war. Umgekehrt hatte längst nicht jeder Ritter eine eigene Burg."

81 Döring, Ritterburg (wie Anm. 78), 1; Stiefenhofer, Ritterburg (wie Anm. 78), 5.

82 Schaller, Burgen (wie Anm. 71), 8-12, $46 \mathrm{f}$.

83 Döring, Ritterburg (wie Anm. 78), 1: „[Burgen aus Stein] konnten besser [als Holzburgen] verteidigt werden. “; Stiefenhofer, Ritterburg (wie Anm. 78), 2: „Er [Eingang zum Bergfried] ist klein, schwer zu erreichen und man kann ihn leicht verteidigen." 
die Belagerung. In der Kindergarten-Reihe wird der Ernst der Belagerungsszene durch abgebildete Tiere und lustige Abbildungen, wie einen Ritter, der in den Wassergraben gestürzt ist und einen Fisch ausspuckt, oder einen anderen, der zu diesem unpassenden Zeitpunkt Wasser lassen muss und in ein Gebüsch rennt, sowie durch Bastelhinweise für einen Schild abgemildert. ${ }^{84}$ Außerdem tragen die Kämpfenden mit einem lächelnden Mund im Gesicht zwar Schild und Waffen, aber keine Rüstungen, wie auf der Seite über das Turnier bzw. über die Vorstellung der Rüstung. Dagegen sind die Münder im Junior-Band - sofern sie überhaupt zu erkennen sind - weit aufgerissen bzw. als waagerechter Strich oder mit einem Emoticon für Trauer dargestellt. ${ }^{85}$ Wenn Kindern dies zu vermitteln ist, sollte darüber nachgedacht werden, ob Kindern in Bezug auf andere historische Aspekte und Zeiten mehr zuzutrauen ist.

Auch Feste und Märkte gehören wie die Belagerung zum Themenspektrum eines Burgenbuchs für jede Altersstufe dazu, was unter anderem den Erfolg der MittelalterMärkte erklärt. Textlängen und -schwierigkeitsgrad nehmen mit dem Alter der Zielgruppen zu. Im Band der WAS IST WAS-Reihe treten auch Personen (z. B. Richard Löwenherz), Jahrhunderte sowie exakte Daten auf. ${ }^{86}$ Außerdem berücksichtigt der Band auch Aspekte der Multiperspektivität, die deutsche Geschichtslehrpläne fordern, da ,Burgen in aller Welt ein Kapitel bilden. ${ }^{87}$ Sachbücher folgen also in manchen Bereichen wissenschaftlichen und didaktischen Impulsen. Die Ritter- und Burgen-Welt hält dabei ein Dilemma bereit, das in der Übung kontrovers diskutiert worden ist: die angemessene Thematisierung und Problematisierung von Geschlechterrollen, die auch in Lehrplänen vorgesehen ist. Waren Mann und Frau im Mittelalter zwar nachweislich nicht gleich und hatten unterschiedliche Rollen zu erfüllen, so ist doch meistens eine verzerrende Selektion in Sachbüchern zu erkennen. Väter, die in den Beispielen fast immer adlige Burgherren darstellen, verheirateten stets ihre Töchter, ohne dass Liebe eine Rolle spielte. ${ }^{88}$ Dass dies auch für Söhne oder die Ritter, die besagte Töchter heirateten, galt, wird jedoch selten explizit erwähnt. ${ }^{89}$ Zwangsehen sind auch ein in Filmen gern verwendetes, die Handlung vorantreibendes Motiv - so z. B. im Pixar-Animationsfilm ,Merida - Legende der Highlands' (Originaltitel: Brave USA/ GB 2012), an dem die Verhandlung moderner Themen wie „Selbstbestimmungsrecht der Frau, Aufkündigung der Familientradition [und] Konflikt mit der elterlichen Autorität" positiv hervorgehoben wurde. ${ }^{90}$

84 Döring, Ritterburg (wie Anm. 78), $9 f$.

85 Stiefenhofer, Ritterburg (wie Anm. 78), $18 f$.

86 Schaller, Burgen (wie Anm. 71), 4f., $18 \mathrm{f}$.

87 Ebd., ab 44.

88 Emmanuelle Lepetit (Text)/Yves Lequesne (Illustration)/Regina Enderle (Übersetzung aus dem Französischen), Weisst Du das? Mittelalter. Fragen und Antworten, Potsdam o. J., 34.

89 Eine der wenigen Ausnahmen bei Karina Trapp, Wir entdecken die Ritterburg (Wieso? Weshalb? Warum?) , 6: „Viele Mädchen und Jungen müssen heiraten, wen ihre Eltern für sie ausgesucht haben - auch wenn er oder sie ihnen gar nicht gefällt."

90 Alexandra Seitz, freie Journalistin und Filmkritikerin, 10.7.2012: https://www.kinofenster.de/film-des-monats/archivfilm-des-monats/kf1207-08/merida-legende-der-highlands-film/ (letzter Zugriff: 26.5.2021). 
Filme und Romane sind nicht geschichtswissenschaftlichen Erkenntnissen verpflichtet. Doch auch in Sachbüchern werden Bilder oder Motive der mittelalterlichen Alltagswelt gebraucht, die in der mediävistischen Quellenanalyse eher selten auftreten und Frauen meistens als Dienerinnen oder Herrin zeigen: Hofdamen und die Herrin ${ }^{91}$ sowie die in Ohnmacht fallende, ${ }^{92}$ eine Kuchen verzierende ${ }^{93}$ und fegende $\mathrm{Frau}^{94}$ sind neben der Frau, die angezogen wird, ${ }^{95}$ die einzigen weiblichen Personen in einem britischen Wimmelbilderbuch, die durch ihre Aufgaben benannt werden. In einer Rettungsszene rettet ein Mann eine Frau. ${ }^{96}$ In den WAS IST WAS-Büchern erscheinen Frauen und Mädchen fast nur in Abbildungen. Eine Märchen-Prinzessin befindet sich zwar auf dem Cover des Kindergarten-Bands, doch ansonsten treten nur die Erzieherin Mona, ${ }^{97}$ eine Wasser schöpfende Magd ${ }^{98}$ und eine junge Dame, die ihren Ritter unterstützt, ${ }^{99}$ auf. ${ }^{100}$ Zwei Abweichungen von der Vorstellung der Frau als Ehefrau, Mutter, höfische Dame, Magd oder Wäscherin sind bei Schaller zu finden: Einerseits wird Uta als Ehefrau des Markgrafen von Meißen gemeinsam mit diesem als Mitbegründende des Naumburger Doms genannt. ${ }^{101}$ Andererseits sei Jeanne d'Arc die einzige bewaffnete Reiterin des Mittelalters gewesen. ${ }^{102}$ Zwar sind Frauen auch in mittelalterlichen Quellen gegenüber den Männern unterrepräsentiert. Aber die Aufgaben von adligen Damen als Fürsprecherinnen, Verantwortliche für das Gebetsgedenken und mitunter Teilhabende an der Herrschaft werden nahezu vollständig ausgeblendet, obwohl Kindern auch heutzutage arbeitende Mütter jenseits von häuslichen Aufgaben vertraut sind. Auch Bilder- und Sachbücher zeigen ein Missverhältnis in der Geschlechterrelation auf, das bei Lesebüchern bereits festgestellt worden ist. ${ }^{103}$ Jedoch sieht die Leseförderung darin eine erfolgversprechende Chance, Jungen zum Lesen zu animieren, ${ }^{104}$ wobei Mädchen nicht zwangsläufig

91 Olivia Brookes (Text)/Nicholas Harris/Sarah Hartley u. a., A year in a Castle, Witney 2012, 2, 5, 7. Laut Verlag ab vier Jahren: http://www.orpheusbooks.com/a-day-a-year (12.2.2020). Dort kann man eine PDF-Version herunterladen.

92 Ebd., 8.

93 Ebd., 6, mit einem Bäcker und einer Bäckerin.

94 Ebd., 10.

95 Reifrock und Schnürung kommen erst im Übergang zur Neuzeit auf; ebd., 6.

96 Ebd., 9.

97 Döring, Ritterburg (wie Anm. 78), 1.

98 Ebd., 3.

99 Ebd., 8; auf dieser Turnier-Doppelseite (7f.) sind 33 Menschen abgebildet, von denen zehn weiblich sind, was das ausgeglichenste Geschlechterverhältnis darstellt; im Junior-Band sind von zwölf abgebildeten Menschen auf der Doppelseite ,Was isst man auf der Ritterburg?' fünf weiblich: Stiefenhofer, Ritterburg (wie Anm. 78), $8 \mathrm{f}$.

100 Döring, Ritterburg (wie Anm. 78). Als Mutter (eines Pagen) bzw. Ehefrau des Burgherrn und Mutter seiner Kinder werden Frauen abgebildet, 5f.; im Junior-Band erscheinen nur Mägde explizit, Stiefenhofer, Ritterburg (wie Anm. 78), 6.

101 Schaller, Burgen (wie Anm. 71), 18. Allerdings nicht im Haupttext, sondern in einer Bildbeischrift.

102 Ebd., 30.

103 Frauenfiguren sind deutlich unterrepräsentiert und häufiger Nebenfiguren vgl. Ylva Schwinghammer, Ritterbuch - Bubenbuch? Neue Ritter(innen) in alten Rüstungen als Akteure einer geschlechtergerechten koedukativen Leseförderung, in: Bennewitz/Schindler (Hrsg.), Mittelalter im Kinder- und Jugendbuch (wie Anm. 56), 138.

104 Sebastian Schmideler, Vom Geöfneten Ritter-Platz (1702) zum postmodernen Jugendroman. Ritterdarstellungen aus vier Jahrhunderten Kinder- und Jugendliteratur, in: Herweg/Keppler-Tasaki (Hrsg.), Rezeptionskulturen (wie Anm. 24), 406f. 
weniger interessiert an diesen Themen seien, obwohl die Ritter-Welt nach wie vor als Jungendomäne vermarktet wird. ${ }^{105}$

Wenn ein Kind dann zum Schulanfang eine kleine Schultüte für kleine Prinzessinnen oder mutige Ritter erhält, ${ }^{106}$ müssen damit nicht zwangsläufig alte Geschlechterrollen zementiert werden. Denn Mädchen sind im Gegensatz zu Jungen eher bereit (gezwungenermaßen?), sich mit Figuren des anderen Geschlechts zu identifizieren. ${ }^{107}$ Dieser Komplex zeigt aber divergierende Haltungen auf, die Erziehungsberechtigte und Lehrpersonen vor Herausforderungen stellt. Durch Werbung vorgegebene Rollen, die Geschlechterunterschiede verstärken könnten, konkurrieren mit persönlichen Erziehungsansätzen und dem Erziehungs- und Bildungsauftrag der Lehrpersonen. Außerdem neigen Bezugspersonen zur Bevorzugung von althergebrachten, bekannten Kinderbüchern bzw. deren Motiven, die ältere Rollen- und Geschichtsbilder nicht nur von Geschlechtern, sondern auch von Familien, der Kirche etc. der 1950er bis 80er Jahre perpetuieren. Vor allem im Bereich der fiktionalen Kinderbücher stellten die Studierenden bei aus den Kinderzimmern mitgebrachten Titeln erstaunt fest, dass diese zum Zeitpunkt der kindlichen Lektüre bereits überholte Gegenwartsdiskurse aufwiesen. Kontrovers wurde diskutiert, ob diese inzwischen mindestens zwanzig Jahre alten Diskurse Kindern heute noch zugemutet werden könnten. Manche Studierende vertraten die Meinung, dass eine Überfrachtung der Kindheit mit Gesellschaftsdiskursen nicht wünschenswert sei.

Insgesamt ist der Eindruck zu gewinnen, dass Kinderbücher im Hinblick auf die genannten Aspekte weniger dynamisch auf gesamtgesellschaftliche Entwicklungen reagieren als etwa Mittelalter-Spielfilme für Erwachsene. ${ }^{108}$ Bei Sachbüchern scheint dagegen ein Umdenken eingesetzt zu haben. Aus mediävistischer Perspektive betrachtet birgt eine Anpassung an aktuelle Gleichstellungsgrundsätze, die manche Kinderbücher im erzählenden Genre mit „einer Umkehrung traditioneller Rollenbilder“109 vertreten, die Gefahr der Schaffung eines ahistorischen Mittelalterbildes. Burgen und Ritter bieten auch nicht den optimalen Anknüpfungspunkt für bedeutende Königinnen oder weibliche Heilige des Mittelalters. ${ }^{110}$ Es gibt daher keinen einfachen Lösungsweg für die Problematik, dass die Vielschichtigkeit mittelalterlichen Gesellschaften zu unterschied-

105 Schwinghammer, Ritterbuch - Bubenbuch? (wie Anm. 103), $143 \mathrm{f}$.

106 Vor ca. 10 Jahren von Lindt vertrieben: Auf der rosa-pinkfarbene Tüte reicht ein schüchtern lächelnder, rosa, aufrechtstehender Bär, der auf der linken Seite steht, einer rosa gekleideten, blonden Prinzessin mit goldenem Krönchen, die leicht zu ihm aufschaut, eine rosa Blume. Im Hintergrund zwischen den beiden ist ein rosa Märchenschloss mit zahlreichen Türmchen und Flaggen zu erkennen, das den Walt-Disney-Themenpark-Schlössern ähnelt. Die rosa Banderole mit der weißen Schrift "Für kleine Prinzessinnen" fällt wasserfallartig von links oben nach rechts unten.

Auf der blauen Tüte ist auf der linken Seite ein grauer, aufrechtstehender Wolf abgebildet, der sich mit dem ihm auf Augenhöhe gegenüberstehenden, jungen Ritter abklatscht. Sie stehen auf der Burgmauer, hinter innen ist der zentrale Bergfried mit blaugelber Fahne zu sehen, auf der ein blaues Wappen mit einem gelben, steigenden Pferd abgebildet ist. Der Junge trägt über seiner Rüstung ein Hemd mit der gleichen Abbildung wie auf der Fahne. Die gelbe Banderole mit der blauen Schrift „Für mutige Ritter" steht horizontal und aufrecht zum Bild.

107 Schwinghammer, Ritterbuch - Bubenbuch? (wie Anm. 103), 139.

108 Lillge, Spielfilme (wie Anm. 22), 509.

109 Schwinghammer, Ritterbuch - Bubenbuch? (wie Anm. 103), 139.

110 Zur Problematik der Darstellung der geistlichen Sphären siehe unten. 
lichsten Zwecken in geschichtskulturellen Erzeugnissen reduziert und manchmal gar karikiert wird. Dadurch wird außerdem - meist implizit - das Fortschritts- bzw. für das Mittelalter sogar Rückschrittsnarrativ der menschlichen Entwicklung weitergeschrieben. Ist es das Ziel des Sachbuchs oder Schulunterrichts, die Überwindung dieser barbarischen Zeit darzulegen, so können die Eigenheiten und Bedeutung(en) des Mittelalters nur unzulänglich vermittelt werden. ${ }^{111}$ So zeigt das Beispiel der Eheschließungen, dass Liebesheiraten im modernen Sinn weder in der Antike noch im größten Teil der Neuzeit der Regelfall waren. Damit ist der Aspekt kein Argument für die Rückständigkeit des Mittelalters und ungeeignet für die Demonstration der Andersartigkeit der Zeit.

Doch wir stellten im Seminargespräch fest, dass die Besprechung von Mittelalterbildern aus verschiedenen Jahrzehnten unter der Anleitung von Lehrpersonen ab der Sekundarstufe I geeignet erscheint, gesellschaftliche Wandlungsprozesse ohne persönliche Überspitzung zu thematisieren und damit einen Raum zum Üben von Diskursen bieten könnte. Das erhöht zwar nicht automatisch das Wissen über aktuelle geschichtswissenschaftliche Mittelalterbilder. Aber die Fähigkeit zu Einordnung von Diskursen in ihren historischen Kontext und das Erkennen von Intentionen der Nutzung dieser Diskurse in der jeweiligen Gegenwart stellen genuine geschichtswissenschaftliche Kompetenzen in einer Wissens- und Informationsgesellschaft dar. Und das allgemeine Interesse am Mittelalter, das Kinder zur Bezeichnung der Epoche als „Ritterzeit“112 verleitet, bietet damit meines Erachtens optimale Voraussetzungen für Lehrpersonen an Schulen, die Fachanforderungen umzusetzen.

\section{Kirche und Gesellschaft}

Die Vielfalt mittelalterlicher Gesellschaften wird im Kindermittelalterbild der Sachliteratur nicht nur in Bezug auf Geschlechterrollen reduziert, sondern auch die Bedeutung der Kirche und des christlichen Glaubens für die Menschen wird nur mit Einschränkungen deutlich. Da die zentralen Gebäude des Mittelalters laut Sachbüchern Burgen bzw. Höhen- und Stadtburgen waren, treten dominierende Kirchengebäude kaum in Erscheinung. ${ }^{113}$ So werden Kirchenbauten, die für Studierende Religion im Mittelalter symbolisieren - allerdings bei deutlich geringerer Nennung als Burgen, ${ }^{114}$ nicht konsequent in dieser Weise für Kindersachbücher genutzt. ${ }^{115}$ Dazu dienen Darstellungen von Personen als Vertreter der Kirche: Manchmal treten einzelne Priester auf Wimmel-

111 So auch Drüding/Enseleit, Ritter (wie Anm. 27), 115.

112 Pape, Geschichtsbewusstsein (wie Anm. 29), 6.

113 Ausnahmen bilden städtische Kathedralen: Schaller, Mittelalter (wie Anm. 43), 36f.: Mitten in der Stadt: die Kathedrale; einzig das umfangreichste Sachbuch mit 127 Seiten hat ein Kapitel "Kirchen überall!", das romanische Kirchen vorstellt und über je ein weiteres Kapitel zu gotischen Kathedralen und Kirchenbaustellen, verfügt Lepetit/Lequesne/Enderle, Weisst Du das? (wie Anm. 88), 96-101.

114 Drüding/Enseleit, Ritter (wie Anm. 27), 109, 112.

115 Eine Ausnahme stellt dar: Oliver Bieber, Klassenbibliothek Mittelalter Niveau 1. Berlin 2010 (Sachunterricht PLUS), 11: Dort steht im Kapitel,Wichtige Gebäude in der Stadt' das Kirchengebäude für die Macht der Kirche über die Menschen. 
bildern auf. ${ }^{116}$ Bei WAS IST WAS werden im Kindergarten-Band weder sakrale Bauten noch Geistliche abgebildet oder erwähnt. Dagegen können etwas ältere Kinder bereits auf der ersten Doppelseite eine Burgdarstellung entdecken, auf der neben dem Bergfried eine Kapelle abgebildet ist. ${ }^{117}$ Es gibt sogar ein Kurzinterview mit einem Mönch, Bruder Gabriel, zum Schulunterricht, wobei der Gegensatz zwischen den Kindern der Adligen, die unterrichtet werden, und Handwerker- und Bauernkindern, die arbeiten bzw. von den Eltern lernen müssen, betont wird. Seine Lehrfähigkeit begründet Gabriel mit seinem Wissen über Religion sowie Lese-, Schreib- und Rechenkompetenzen. ${ }^{118}$

Ein Bischof erscheint erst für Grundschüler*innen wiederum in Verbindung mit gesellschaftlichen Unterschieden: Das Lehnswesen wird mit einer Ständepyramide illustriert, in der Herzog und Bischof auf der gleichen Stufe unterhalb des Königs als Personen stehen. ${ }^{119}$ Direkt unter der Pyramide befindet sich ein Info-Kasten zu Besitzverhältnissen, die unter dem Lehnswesen firmieren, wobei eine Verschränkung mit der Grundherrschaft erkennbar ist, die aber nicht benannt wird. Eine Doppelseite des Oberkapitels ,Leben auf der Burg' ist dem ,Beten, fasten und feiern' gewidmet und erklärt die Allgegenwart des christlichen Glaubens im Mittelalter: Burgen hatten Kapellen und Priester waren bei allen Festen, auch bei Siegesfeiern und Schwertleiten, anwesend, wobei die Nennung des von der Kirche etablierten freien Sonntags eher als Durchgangsstation zum Sonntagsbraten und damit zu Festmählern auf der folgenden Seite als zur Darlegung christlicher Frömmigkeit dient. ${ }^{120}$ Außerdem wird das hart arbeitende Personal der feiernden Herrschaften hier und bei der Nennung der notwendigen Arbeiten auf einer Burg gegenübergestellt. ${ }^{121}$ Dass Feste im Mittelalter selten der reinen Unterhaltung, sondern oft einem konkreten Anlass dienten, wie etwa dem Abschluss von Verhandlungen jedweder Art oder der Repräsentanz und Bestätigung des Status der Teilnehmenden, wird ebenso wie die bewusste Terminierung auf hohe Feiertage ${ }^{122}$ in Sachbüchern nicht ausgeführt. Frömmigkeit wird nur mit einem tiefen Glauben an Gott erklärt: Auch Frauen sollten häufig beten und beichten, wozu die Burgkapellen dienten, die in größeren Bauten die soziale Hierarchie mit oberer und unterer Kapelle demonstrieren konnten. ${ }^{123}$ Frömmigkeit erscheint hier als institutioneller Zwang, der Laienfrömmigkeit völlig ausblendet. Außerdem kann die mittelalterliche Würdigung von Frauen beim Gebetsgedenken in dieser Form der Darstellung keine Entsprechung finden.

\footnotetext{
116 Brookes, A year in a Castle (wie Anm. 91), 5.

117 Stiefenhofer, Ritterburg (wie Anm. 78), 2.

118 Ebd., 4.

119 Schaller, Burgen (wie Anm. 71), 7. Auf der nächsten Stufe folgt der Ritter und zwischen diesem und den auf dem Boden stehenden Bauern befindet sich noch ein Mönch.

120 Ebd., 24-26.

121 Ebd., 20-23.

122 Ebd., 24f. Die Kirche habe sie festgelegt und einige werden aufgezählt.

123 Ebd., 25.
} 
Im Band zu Rittern wird auf vier der 46 Seiten der Glaube eines Ritters und die Kreuzzüge, die in den Fachanforderungen genannt werden, thematisiert. ${ }^{124}$ Die Kreuzzüge haben ein gemeinsames Oberkapitel mit dem Ende des Mittelalters am Beispiel der Pest im Mittelalter-Band. ${ }^{125}$ Dieser Band ist der einzige Band der WAS IST WASReihen, der auf das Klosterwesen am Beispiel von Nonnen und Mönchen eingeht. ${ }^{126}$ Außerdem werden bereits in einem Einstiegsabschnitt ,Glaube und Schicksal ${ }^{\star}$ als Teil der mittelalterlichen Welt eingeführt, der wieder auf ,Gesellschaft und Ständepyramide‘ hinführt. ${ }^{127}$ Auch wenn die Burgherrin „viel zu tun“ ${ }^{128}$ hatte und der Burgherr „so etwas wie Großbauer, Bürgermeister, Richter, Polizeipräsident und Heeresführer in einem“129 war, scheint nur das „fleißige Gesinde [...] sich den Rücken krumm“ ${ }^{130} \mathrm{zu}$ arbeiten. Bei Bauern wird häufig - vor allem in französischen Büchern oder bei der Thematisierung von Frankreich - die hohe Abgabenlast betont. ${ }^{131}$ Schon in Kinderbüchern sind also nationale Mittelalterschwerpunkte zu erkennen.

Die Kreuzzüge können in der Sekundarstufe I als Beispiel für ,Juden, Christen und Muslime: Das Mittelalter - eine finstere Zeit? genutzt werden. ${ }^{132}$ In der Übung haben wir im Hinblick auf die Thematisierung der Kreuzzüge festgestellt, dass multiperspektivische Ansätze der Darstellung in Sachbüchern noch oft verbesserungswürdig sind. ${ }^{133}$ Im Bezug auf nicht-eurozentrische Narrative von muslimisch geprägten Schülerinnen und Schülern ist dieser Punkt von Relevanz und bedarf noch eingehender Analysen. Ob die in der Sekundarstufe II zum Feudalismus zu diskutierende Frage, ob Grundherrschaft eher Unterdrückung und Ausbeutung oder Schutz und soziale Fürsorge gewesen sei, ${ }^{134}$ ein differenzierteres Bild ergibt als die Präkonzepte der Kinderbücher, dürfte stark von der Qualität des Unterrichts und der Aufnahmebereitschaft der Schüler*innen abhängen, zumal Schulbücher eher das institutionelle Kirchenbild fördern. ${ }^{135}$

\footnotetext{
124 Andrea Schaller, Ritter. Burgen, Turniere, edle Frauen. Nürnberg 2014 (WAS IST WAS, Bd. 88), 40-43.

125 Dies., Mittelalter (wie Anm. 43), 42-47: ,Die Kreuzzüge und das Ende des Mittelalters' mit den Unterkapiteln ,Mittelalter im Galopp', , Jerusalem - der Nabel der Welt' und ,Wirklich die Pest: das 14. Jahrhundert'.

126 Ebd., 32f.

127 Ebd., 14-17: Kapitel ,Glaube und Schicksal' und ,Gesellschaft und Ständepyramide'.

128 Schaller, Burgen (wie Anm. 71), 20.

129 Ebd., 19.

130 Ebd., $22 f$.

131 Jean Petit/Cécile Marais, Der grosse Wimmel-Suchspaß. Entdeckungsreise durch die Geschichte. In Ägypten. In Gallien. Im Mittelalter, aus dem Französischen von Christine Gallus, München 2004, 69: „Sie [die Bauern] müssen diesem [dem Burgherren] einen Großteil ihrer Ernte und ihres Viehs abgeben.", oder Lepetit/Lequesne, Weisst Du das? (wie Anm. 88), 64: „Für die Bauern waren sie [Frondienste] eine große Last [...]. Sie wurden rücksichtslos eingefordert.", oder Timothy Knapman (Author)/Wesley Robins (Illustrator), Let's go back in History. Dig millions of years through time! Bicester 2008, [Kapitel] Medieval France. 800 Years ago: „Ordinary people had to work hard on thin strips of land to grow enough food to eat."

132 Leitfaden zu den Fachanforderungen Geschichte (wie Anm. 50), 16.

133 Beispielsweise richtet nur eine Frage im Kapitel ,Kreuzzüge' (S. 104-107) in Lepetit/Lequesne, Weisst Du das? (wie Anm. 88), den Blick auf die Gegner mit abwertenden Attributen: „Warum waren die arabischen Krieger so schrecklich?", 106.

134 Fachanforderungen Schleswig-Holstein (wie Anm. 50), 29.

135 Drüding/Enseleit: Ritter (wie Anm. 27), 112. Vgl. dort die weiterführenden Literaturhinweise.
} 
Dass Kirche und ihre Vertreter gar nicht oder fast nur in Verbindung mit Herrschaft und Krieg in Kinder-Mittelalterbildern erscheinen, dürfte zum negativen Kirchenbild von Jugendlichen und Studierenden beitragen. Außerdem bietet die Geschichtskultur wenig Anhaltspunkte dafür, was die sakrale Kultur des Mittelalters sein könnte. Wenn diese Frage also auch in Schulen nicht intensiv thematisiert wird, ist es nicht erstaunlich, dass Studierende die Aussage, dass das Mittelalter eine sehr religiöse Zeit gewesen sei, als Ergebnis und nicht als Ausgangspunkt einer Untersuchung betrachten.

Die Religiosität des Mittelalters wird von Studierenden in der Regel negativ bewertet und einer machthungrigen Kirche angelastet. ${ }^{136} \mathrm{Ob}$ die Gründe dafür in einer immer noch wirksamen, protestantischen Propaganda gegen die Papstkirche des 16. Jahrhunderts, einem zunehmenden Bedeutungsverlust der Kirchen in säkularen Staaten oder der Übertragung negativer Bewertungen religiös motivierter Handlungen und Politik in der Gegenwart zu suchen sind, wäre noch zu untersuchen. Jedenfalls haben Untersuchungen an Schulen gezeigt, dass die Vorstellung, die mittelalterliche Kirche habe intentional aus ökonomischen Gründen die Gläubigen getäuscht und die Herrschaft von Mächtigen gestützt, verbreitet ist. ${ }^{137}$

Auch die Verwendung von Bibelbezügen in der mittelalterlichen Literatur wird in Kinderbüchern kaum berücksichtigt, da auf die höfische Minne maximal durch Bildzitate aus dem Codex Manesse eingegangen wird. ${ }^{138}$

Diese Befunde machen deutlich, dass diese Präkonzepte geeignet erscheinen, Grundlagen für Vorstellungen zum Mittelalter zu legen, die auch bei Schüler*innen und Studierenden sowie in der Gesamtbevölkerung verbreitet sind. ${ }^{139}$ Daraus folgt nicht zwangsläufig, dass die Darstellungen von Kinder-Sachbüchern für diese verantwortlich sind. Jedoch scheinen diese Präkonzepte vom Mittelalter extrem stabil und resistent zu sein, ${ }^{140}$ sodass sie nicht nachhaltig vom Geschichtsunterricht verändert werden. Gelingt es auch der Hochschullehre nicht, diese zu diskutieren und nutzbar zu machen, besteht die Gefahr, dass zukünftige Lehrkräfte erneut diese Wahrnehmungen tradieren und reproduzieren werden.

136 Wobei oftmals wenig Wissen zum Christentum damals und heute vorhanden ist.

137 Friederike Stöckle, „Die armen kleinen Bäuerlein...”. Schülervorstellungen zu mittelalterlichen Herrschaftsformen. Ein Beitrag zur Didaktischen Rekonstruktion. Oldenburg 2011 ((Beiträge zur Didaktischen Rekonstruktion, Bd. 35), 189, 260f.

138 Schaller, Burgen (wie Anm. 71), 35; Graf Kraft von Toggenburg: Codex Manesse, BL. 22v; auch bei Lepetit/ Lequesne, Weisst Du das? (wie Anm. 88), 32f., fehlt jeglicher geistliche Bezug.

139 Drüding/Enseleit, Ritter (wie Anm. 27), 109; eine ältere und in manchen Teilen aufgrund neuer Medien vielleicht auch nicht mehr aktuelle Studie bietet Bodo von Borries, Das Geschichtsbewußtsein Jugendlicher. Eine repräsentative Untersuchung über Vergangenheitsdeutungen, Gegenwartswahrnehmungen und Zukunftserwartungen von Schülerinnen und Schülern in Ost- und Westdeutschland. Weinheim/München 1995 (Jugendforschung), 56-60, wobei Hexenverfolgungen häufiger genannt werden als bei meinen Umfragen; Stöckle, „Die armen kleinen Bäuerlein...". Schülervorstellungen (wie Anm. 137).

140 Zur Resistenz und Stabilität von Präkonzepten Franziska Streicher/Monika Fenn: Modifikation von Präkonzepten über den Konstruktcharakter von Geschichte. Skizze einer explorativen Interventionsstudie in der Primarstufe, in: Fenn, Lernen (wie Anm. 26), 204. 


\section{Kindermittelalterbilder bei Studierenden und Mittelalter in den Fachanforderungen}

Burgen und Ritter können als gesamtgesellschaftliches Kindermittelalterbild beeinflusst durch Werbung, Spielzeug etc. und vor allem Bezugspersonen vorausgesetzt werden. Sie sind Dreh- und Angelpunkt für das Mittelalterbild, da fast alle anderen Themen über sie in Beziehung gesetzt werden: Ritter konnten auch Kreuzritter sein, die Hygiene auf Burgen war nicht besonders ausgeprägt, weil „Badespaß“141 ein seltener Luxus war. Insofern bilden Ritter und Burgen das einzige omnipräsente Mittelalterbild bei Kindern. Doch aus der Begeisterung für Ritter-Tütensuppen oder andere geschichtskulturelle Erzeugnisse resultiert nicht automatisch ein anhaltendes Interesse am Mittelalter oder der Wunsch nach einem Geschichtsstudium mit einem mittelalterlichen Schwerpunkt, worauf einzelne Studierende im Hinblick auf den eigenen Werdegang hinwiesen. ${ }^{142}$

Diese Anregung wurde im Wintersemester 2019/20 anhand eines anonymen Fragebogens aufgenommen. Er sollte Hinweise zur Frage liefern, wie stark die Verbreitung der Erfahrung mit mittelalterlicher Geschichtskultur ist und ob die Studierenden diesen Einfluss auf das eigene Interesse anerkennen. Ausgewertet wurde die nicht repräsentative Umfrage in einem Proseminar (18 Teilnehmende) und einer Methodikübung zum Kinder-Infotainment (neun Teilnehmende). 25 von 26 Studierenden gaben an, dass sie vorschulische oder aus der Grundschule (nur insgesamt acht) resultierende Erfahrungen mit Mittelalterbildern haben - und zwar mit Rittern. Empirische Erhebungen der Geschichtsdidaktik sprechen sich für eine Verstärkung des Geschichtsanteils des Sachkundeunterrichts an Grundschulen aus, ${ }^{143}$ wobei der Unterricht aber nicht allein vorhandenes Wissen reproduzieren dürfe. ${ }^{1 / 4}$ Der explizite Mittelalterteil der frisch in Kraft getretenen Fachanforderungen in Schleswig-Holstein ist inzwischen gegenüber dem vorherigen Lehrplan, der noch Haithabu, Stadt, Land und Siedlungspuren als möglichen Mittelalterbezug für Klasse 4 verzeichnete, gleich null. ${ }^{145}$ Zwar könnten unter dem Aspekt ,Zeit und Entwicklung mittelalterliche Themen behandelt werden. Allerdings soll in den meisten deutschen Lehrplänen zu diesem Thema rudimentäres Zeitbewusstsein anhand der Uhr behandelt werden. ${ }^{146}$ Laut Bildungsplan Sachunterricht für Hamburg gilt die Regelanforderung am Ende der 4. Klasse, dass Schüler*innen Epochen - u. a. das

\footnotetext{
141 Schaller, Burgen (wie Anm. 71), 21.

142 Ein Beispiel dafür ist die Verfasserin, die keine Spielzeug-Ritterburg oder themenverwandte Bücher in ihrer Kindheit besaß. Mit Beginn des Geschichtsunterrichts war zwar ein Grundstein für das Geschichtsstudium gelegt, aber das Interesse für das Mittelalter kam erst mit den ersten Mittelalter-Proseminaren in Geschichte und Älterer deutscher Literatur.

143 Pape, Geschichtsbewusstsein (wie Anm. 29), 1.

144 Christian Mathis/Ludwig Duncker, Historisches Lernen im Sachunterricht. Perspektivwechsel als Merkmal der Unterrichtsqualität, in: Fenn, Lernen (wie Anm. 26), 92.

145 Ministerium für Bildung, Wissenschaft, Forschung und Kultur des Landes Schleswig-Holstein (Hrsg.), Lehrplan: Grundschule: Heimat- und Sachunterricht, Kiel o. J., 107; Ministerium für Bildung, Wissenschaft und Kultur des Landes SchleswigHolstein (Hrsg.), Fachanforderungen Sachunterricht Primarstufe/ Grundschule, Kiel 2019.
}

146 Becher/Gläser, Präkonzepte (wie Anm. 59), 85. 
Mittelalter - benennen können sollen. ${ }^{147}$ Das ,Leben im Mittelalter ${ }^{`}$ zählt dort zu den verpflichtenden Inhalten, wobei es durch „seine Ritter und Burgen und den verschiedenen Handwerksberufen" den Schüler*innen in der Geschichtskultur begegne und damit die Kompetenzentwicklung in der historischen Perspektive besonders fördere. ${ }^{148}$ Lehramtsstudierende der Geschichte für die Primarstufe müssen an der Universität Hamburg inzwischen aber nicht mehr verpflichtend eine Veranstaltung in der mittelalterlichen Geschichte absolvieren. ${ }^{149}$ Sollten die Absolvent*innen in Hamburg bleiben, hängen ihr Wissen und ihre Kompetenzen also sehr stark von ihrer Eigeninitiative oder dem an Schulen vorliegenden Material ab, weil es wenig Schulbücher für Grundschulen mit dem Schwerpunkt Mittelalter gibt. ${ }^{150}$ Außerdem bieten diese keine Hilfe zum Aufbau elaborierter Konzepte zur Ablösung von Präkonzepten, die in der Grundschule geboten werden müssten. ${ }^{151}$

Damit können Grundschulen die durch die Förderung von Bezugspersonen entstandenen Wissens-(,weniger Kompetenz-)Unterschiede im Bereich der mittelalterlichen Geschichte kaum kompensieren, auch wenn es sich um ein eingeschränktes Repertoire an Themen handelt. Das Phänomen, dass Kinder- und Jugendliteratur (bzw. die nachfragenden Personen) Wissensunterschiede aufgrund sozialer Herkunft verstärken können, ist nicht gänzlich neu. Schon für Kinder- und Jugend-Bildungsbücher des 18. und 19. Jahrhunderts ist dies nachzuweisen. ${ }^{152}$

Die weiteren Ergebnisse des Fragebogens deuten an, dass das Mittelalterwissen von Studierenden zu einem erheblichen Teil auf Kinder-Mittelalterbildern beruhen dürfte. Denn auf die Frage, ob und welche Mittelalterthemen in der Sekundarstufe I behandelt wurden, antworteten vier, dass sie es gar nicht thematisiert hätten. Zehn konnten sich nicht mehr an die Inhalte erinnern. Nur knapp die Hälfte der Befragten konnte Beispiele nennen (13), wobei ,Stände ' mit sechs Mal am häufigsten genannt wurden. Je drei Mal wurden Karl der Große sowie Staat und Kirche, je einmal Essen, Krankheiten, Kreuzzüge und Kurfürsten genannt. ${ }^{153}$

147 Freie und Hansestadt Hamburg, Behörde für Schule und Berufsbildung (Hrsg.), Bildungsplan Grundschule. Sachunterricht, Hamburg 2011, 21.

148 Ebd., 26.

149 Seit dem WS 2014/15 gelten die Studienanforderungen des Unterrichtsfachs Geschichte B.A.-Lehramt Primar- und Sekundarstufe I, die ein Proseminar plus Vorlesung in der Vormoderne verlangen und die Möglichkeit bieten, eine Übung für das Epochenmodul oder ein Hauptseminar im Bereich Mittelalter für das Vertiefungsmodul zu wählen. Allerdings besteht die Möglichkeit, ohne mittelalterliche Lehrveranstaltung das Studium zu absolvieren. Der Verlaufsplan für das 1. Staatsexamen von 1999 sah noch obligatorische Proseminare und Vorlesungen sowohl für die Alte als auch für die Mittelalterliche Geschichte sowie ein obligatorisches Hauptseminar in einer der beiden vormodernen Epochen vor.

150 Beispielsweise bei Cornelsen: Bieber, Mittelalter (wie Anm. 115); Anne Scheller, Rund um Ritter. Kopiervorlagen für den Unterricht an Grundschulen (2.-4. Schuljahr), Berlin 2009, wobei Ritter nur die Szenerie bieten. Digitales Lehrmaterial im Internet orientiert sich an Ständepyramiden und ähnlichen bekannten Themen: https://lehrermarktplatz.de/material/149297/ mittelalter-burg-burgbewohner-ritter (letzter Zugriff: 26.5.2021).

151 Am Beispiel des Konstruktcharakters von Geschichte, vgl. Streicher/Fenn, Modifikation (wie Anm. 140), 204.

152 Schmideler, Ritterdarstellungen (wie Anm. 104), 384-395; zur Funktion der mittelalterlichen Geschichte als Elitencurriculum an Universitäten des 19. und beginnenden 20. Jahrhunderts vgl. Julien Demade, The Contemporary Delegitimization of (Medieval) History - and of the Traditional, University Curriculum as a Whole, in: Jones/Kostick/Oschema (Hrsg.): How Medieval Studies (wie Anm. 5), 143-146.

153 Auch Hexenverbrennungen und Buchdruck wurden als Zäsurmerkmale zur Neuzeit und Nathan der Weise oder Die Buddenbrooks, die in die Neuzeit gehören, genannt. 
In der Sekundarstufe II ist mittelalterliche Geschichte seltener Unterrichtsgegenstand. Zehn Studierende haben kein Mittelalter im Unterricht thematisiert, drei sind sich nicht sicher, ob sie es vergessen haben, fünf haben mittelalterliche Themen behandelt, ohne sich erinnern zu können. Nur zehn Personen können sich an Inhalte erinnern: Stände und Gesellschaft, Tempelritter und Kreuzzüge (mit der Betonung: „beide Seiten“), jüdisches Leben und seine Bedrohung, Chlodwig, Pest und Hanse im Fach Geschichte. Also sollten die Möglichkeiten zum Aufbrechen des chronologischen Schulunterrichts, die heutige Fachanforderungen bieten, genutzt werden, um mittelalterliche Themen präsenter zu machen. Das bedeutet für Hochschullehrende, dass das Thematisieren eines für Querschnittsthemen geeigneten Beispiels in Lehrveranstaltungen bedenkenswert ist.

Obwohl der Schulunterricht des Faches bei der Mehrheit der Studierenden nicht als förderlich angesehen wird, stufte die Mehrheit ihr Interesse an mittelalterlicher Geschichte als hoch ein: ${ }^{154}$ Sie beschäftigten sich mit Geschichtskulturen in Museen, Dokumentationen, Romanen, digitalen Spielen - und auch die Serie ,Games of Thrones wurde mehrfach genannt. Die meisten Studierenden des Proseminars äußerten sich jedoch skeptisch gegenüber der universitären Beschäftigung mit Geschichtskultur. Dabei handelt es sich vermutlich um einen Ausdruck der Sorge oder Unsicherheit, ob die Geschichtswissenschaft eine Thematisierung gutheißen würde. Meine These dazu ist, dass es sich um einen Versuch der habituellen Professionalisierung handelt, der vor allem zu Beginn des Studiums zu beobachten ist. Er dürfte außerdem eine Folge der geringen geschichtswissenschaftlichen Beschäftigung mit mittelalterlicher Geschichtskultur sein.

Die Studierenden der Methodikübung, die den Kurs alle aus persönlichem Interesse gewählt hatten und mindestens im 5. Semester studierten, zeigten in ihren Antworten ein deutlich höheres Reflexionsniveau, was einerseits mit dem Interesse und anderseits mit Erfahrungen aus Grundschulpraktika zusammenhängen dürfte.

Aber Geschichtskultur vermittelt offenbar Interesse, das Lehrkräften in der Schule wieder begegnen wird. Die im Bereich der Naturwissenschaften bereits übliche Berücksichtigung der Ausgangslage der Lernenden sollte auch bei der Förderung historischer Kompetenzen berücksichtigt werden. ${ }^{155}$ Eine Ablehnung geschichtskultureller Erzeugnisse, die diese Ausgangslage bilden, könnte das Interesse der Schüler*innen erlahmen lassen und die angestrebte Veränderung von Präkonzepten unterlaufen. Im ungünstigsten Fall würde damit ein Grundstein für eine Wissenschaftsskepsis gelegt. Daher sollte die geschichtswissenschaftliche Mediävistik Lehramtsstudierende auf einen angemessenen Umgang mit Geschichtskultur vorbereiten, damit gar nicht erst die Basis für die Wahrnehmung eines widersprüchlichen und unüberwindbaren Gegensatzes zwischen allgemeinen und wissenschaftlichen Mittelalterbildern gelegt wird. Das bedeutet für

154 Dabei ist nicht zu prüfen, ob es sich um tatsächliche oder sozial erwünschte Antworten handelt.

155 Zabold, Ausprägungen historischen Denkens (wie Anm. 60), $61 \mathrm{f}$. 
die mediävistische Hochschullehre, dass sie diskutieren sollte, welche wissenschaftlichen Geschichtsbilder sie vermitteln will und wie das unter Berücksichtigung von Geschichtskultur erreicht werden kann. Außerdem können bei der Lehrplanung die Fachanforderungen berücksichtigt werden, um Anknüpfungspotentiale zu bieten.

\section{Fazit}

Die grundsätzlichen Vorannahmen waren also zutreffend, bedürfen in der Breite aber noch der detaillierteren Überprüfung nach Gattungen (digital und analog), Altersgruppen, Korrelation und Kausalität sowie tatsächlichem Einfluss auf die Wahrnehmung der Zeit: Kinder-Mittelalterbilder stellen mit sehr viel Rittern und Burgen und wenig Kirche, Christentum oder Frömmigkeit ein sehr eingeschränktes Spektrum der wissenschaftlichen Mittelalterbilder dar, wobei altersgemäße Abstufungen zu erkennen sind, zu deren Entstehung die Mediävistik bisher keinen Beitrag geleistet hat. Deshalb fragte ich in beiden Veranstaltungen bei verschiedenen Aspekten die Studierenden nach ihren Vorschlägen für eine geschichtswissenschaftlich angemessene Darstellung, die Kinder als Zielgruppe altersgemäß berücksichtigt. Am Beispiel der statischen Kirchendarstellung wurde festgestellt, dass die Abbildung von Kirchen unterschiedlicher Größen und Baustile mit verschiedenen Vertretern der Kirchenhierarchie sowie Mönchen in der Kleidung der unterschiedlichen Orden auf plakative Weise sogar Kindergarten-Kindern zeigen könne, dass die Kirche im Mittelalter vielgestaltig war. Diese Vorgehensweise stellt eine Möglichkeit dar, unsere Fähigkeiten der didaktischen Reduktion zu üben und ein Angebot für die Verbindung von allgemeinen und fachwissenschaftlichen Mittelalterbildern ${ }^{156}$ zu schaffen. Hochschuldidaktisch sollten wir die Vermittlungstechniken und -methoden solcher Ansätze prüfen, da die beschriebenen Präkonzepte so stabil sind, dass sie selbst bei Studierenden noch häufig nachzuweisen sind. Die Beschäftigung mit Kinder-Mittelalterbildern lohnt sich also für Dozierende, damit sie einerseits wissen, welchen Erwartungen sie ggf. entgegentreten müssen. So bietet der Diskurs zu Geschichtskulturen in der Hochschullehre die Möglichkeit, dass die Studierenden ihr eigenes Geschichtsbewusstsein und ihre Mittelaltervorstellungen hinterfragen. Die Analyse von Kinder-Mittelalterbildern öffnet einen kritischen Blick auf die eigene Abhängigkeit von diesen. Dadurch lässt sich die Veränderungsresistenz von solchen Konzepten als Lernhürden ${ }^{157}$ zur Selbstreflexion sichtbar machen und ein Anwendungsbeispiel für angehende Lehrkräfte an Schulen durchspielen. Andererseits übt die Überprüfung von (klischeebeladenen) Präkonzepten geschichtswissenschaftliches Wissen und Kompe-

156 Zum Beispiel des historischen Erkenntnisgewinns vgl. Monika Fenn, Conceptional change von Vorstellungen über epistemologische Basiskonzepte bei Grundschülerinnen und -schülern fördern? Ergebnisse einer Interventionsstudie, in: Dies. (Hrsg.), Lernen (wie Anm. 26), 146-199.

157 Drüding/Enseleit, Ritter (wie Anm. 27), 114; Streicher/Fenn, Modifikation (wie Anm. 140), 204; Stöckle, „Die armen kleinen Bäuerlein...". Schülervorstellungen (wie Anm. 137), 298. 
tenzen ein und kann einen Beitrag zur Vertiefung dieser historischen Kompetenzen bei Studierenden leisten. Denn Studierende, die Mittelalterbilder in ihrem historischen Kontext analysieren und bewerten können, sind auch in der Lage, die gleichen Kompetenzen und Prozesse auf Quellen und Forschungsliteratur anzuwenden. Außerdem können didaktisch-reduzierte Ansätze der Rezeptionsgeschichte des Mittelalters im Schulunterricht nutzbar gemacht werden, um Kompetenzen zu historischen Diskursen und Narrativierung an besonders beliebten Beispielen zu üben. Darüber hinaus eröffnet diese Herangehensweise die Möglichkeit des Vergleichs von wissenschaftlichen und nicht-wissenschaftlichen Geschichtsbildern zur Feststellung der Unterschiede und Gemeinsamkeiten: Studierende werden in die Lage versetzt, ihre Arbeitsprozesse und die damit verbundenen Aufgaben in gesamtgesellschaftlichen Zusammenhängen zu reflektieren und zu vertreten. Damit eröffnen wir die Möglichkeit, einen ,Graswurzel'-Beitrag zur Kommunikation zwischen Geschichtswissenschaft und Gesellschaft zu leisten.

Außerdem dient die Analyse von Geschichtsbildern der Qualifizierung der Studierenden auch im Hinblick auf spätere Berufe: In klassischen Berufsfeldern wie beispielsweise Museen sind Kinder zwecks Gewinnung zukünftiger Besucher*innen eine wichtige Zielgruppe, die mit explizit für sie (bzw. die Bezugspersonen) gestalteten Programmen angesprochen wird. Wissen und Fertigkeiten im Bereich von Kindermittelalterbildern und rezeptionsgeschichtlichen Perspektiven sind also gefragt.

Schließlich fördern diese Fähigkeiten auch die Qualifizierung der Lehramtsstudierenden: Aus historisch-strukturellen Gründen studieren an der CAU zwar ausschließlich angehende Lehrkräfte für die Sekundarstufe I und II, sodass die Beteiligung an der Diskussion zur mittelalterlichen Geschichte an Primarschulen nur eingeschränkt beim verpflichtenden Grundschulpraktikum möglich ist. Allerdings bilden Kinder-Mittelalterbilder den Grundstock für das Wissen der Schüler*innen in der Sekundarstufe I, sodass mehrere Gründe für eine Thematisierung der Geschichtskultur bereits im Studium sprechen: Erstens steht mit dem Aspekt „Lernen am anderen Ort (z. B. „Mittelalter-Märkte“) “158 Geschichtskultur im Lehrplan, sodass eine geschichtswissenschaftliche Beschäftigung damit sinnvoll ist, um Theorie und Praxis zu verbinden. Zweitens wird damit der Weg für die von Buck geforderte „engere institutionelle Zusammenarbeit zwischen Universitäten, der Geschichtsdidaktik und den Schulen“159 bereitet, indem sowohl das Bewusstsein für die Notwendigkeit als auch Wissen und Kompetenzen im Bereich verschiedener Mittelalterbilder für Lehrkräfte an Schulen und Hochschulen geschaffen wird, die die Zusammenarbeit erleichtern wird. ${ }^{160}$ Idealerweise fördern diese Maßnahmen drittens die Fähigkeiten der Schüler*innen, historische Diskurse bzw. die

158 Fachanforderungen (wie Anm. 50), 20.

159 Buck, Mittelalter (wie Anm. 12), 41.

160 Dazu hat auch das, Geschichtsdidaktische Forschungskolloquium: Geschichtsdidaktische Perspektiven auf die Vormoderne', in dessen Rahmen dieser Beitrag gehalten, angeregt kommentiert und diskutiert wurde, einen Beitrag geleistet, der zur Wiederholung einlädt. 
Rezeption und den Gebrauch bzw. Missbrauch derselben erkennen, einordnen und bewerten zu können. Für gesamtgesellschaftliche (, nicht nur historische) Diskurse einer Wissens- und Informationsgesellschaft stellt dies ein wünschenswertes Ziel dar. Dadurch könnte der Multiplikator-Weg von Hochschullehrveranstaltungen über Lehramtsstudierende in die Schulen einen Beitrag zur indirekten Kommunikation zwischen Geschichtswissenschaft und Gesellschaft leisten: Aufgaben, Methoden und Ziele der mittelalterlichen Geschichtswissenschaft können dann auch außerhalb des vermeintlichen Elfenbeinturms wahrgenommen und wertgeschätzt werden.

Im Hinblick auf die Erreichung dieses Ziels sollten die Möglichkeiten, die die meisten Fachanforderungen zum Aufbrechen des chronologischen, den Fortschritt der Menschheit dokumentierenden Lernens, bieten, konsequent und ohne Furcht vor vermeintlichem Wissensverlust ${ }^{161}$ genutzt werden. Diesbezüglich ist die geschichtswissenschaftliche Mediävistik gefragt für die Planung der Hochschullehre auch die Fachanforderungen und die sich daraus ergebenden Möglichkeiten für Themen zu berücksichtigen, um angehenden Lehrkräften die Chance zu geben, sich bereits im Studium ein Themen- und Methodenset dafür zu erarbeiten.

Als Desiderate stellten sich schließlich sowohl europäisch-vergleichende Perspektiven als auch die Zusammenarbeit von Geisteswissenschaften und Naturwissenschaften heraus: Französische Kinderbücher weisen eine starke Betonung des ungerechten Feudalismus auf. Eine Überprüfung der nationalen Präkonzepte auch von Schüler*innen, die noch vollkommen unerforscht sind, da Schule und Bildung in Form von Fachanforderungen (Bundes-)Ländersache ist, könnte interessante Beiträge zu einem europäischen Dialog bieten. Die Forschung zum Einfluss von digitalen Methoden und Techniken in der Schule ist noch relativ neu. Der diachrone Vergleich von Kindersachbüchern zeigte Veränderungen in der Gestaltung auf, die auch eine Folge von Digitalisierungstendenzen sein könnten, wobei das Verhältnis von Korrelation und Kausalität noch zu klären ist. Auch das Mittelalter kennt zahlreiche technische und mediale Neuerungen, die in das allgemeine Mittelalterbild nur mit dem Ende der Zeit durch den Buchdruck eingegangen sind. Spezialisten*innen des Mittelalters könnten die Diskussion zur Schule der Zukunft als Chance zur Beteiligung an einer gesamtgesellschaftlichen Debatte nutzen, welche die Vielseitigkeit der Mittelalterbilder aufzeigt.

161 Der Wissensverlust, der oben auch schon als Entrümpelung positiv bewertet wurde, wäre freilich noch nachzuweisen, zumal vorher zu klären ist, ob das Wissen, dem die Allgemeinheit (Eltern, Großeltern, Lehrkräfte, Vorsitzende von Verbandsund Lobbyorganisationen etc.) nachtrauert und welches vor Jahren erworben wurde, dem aktuell gesicherten Wissen der Geschichtswissenschaft entspricht. Die weite Verbreitung falscher Mittelalterbilder lässt diesbezüglich wenig Hoffnung. 


\section{Autorin}

\section{Janina Lillge}

Wissenschaftliche Mitarbeiterin am Historischen Seminar der Christian-Albrechts-Universität zu Kiel. Ihre Schwerpunkte liegen in der Theorie und Praxis der historischen Grundwissenschaften und den Einsatzmöglichkeiten von ,Medievalism' in der (Hochschul-) Lehre sowie der früh- und hochmittelalterlichen Geschichte der Verwandtschaft.

lillge@histosem.uni-kiel.de

\section{Anhang}

Fragebogen (18 Teilnehmende im Proseminar / 9 Teilnehmende in der Übung)

Bitte beantworten Sie folgende Fragen (ggf. auch mit „Ich erinnere mich nicht.") oder kreuzen das Zutreffende an.

- Haben Sie Erfahrungen mit dem Mittelalter gemacht, bevor Sie eingeschult worden sind, an die Sie sich erinnern können? Wenn ja, nennen Sie diese und die vermittelnden Personen und Zusammenhänge.

- Haben Sie in der Grundschule das Mittelalter behandelt? Wenn ja, nennen Sie bitte das Thema und ggf. Zusammenhänge.

Ergebnis: 25 von 26 antworteten mit ,ja' (ausschließlich Ritter und Burgen sowie Erziehungsberechtigte; das gleiche Thema haben acht in der Grundschule behandelt).

- Haben Sie in der Sekundarstufe I das Mittelalter behandelt? In welchem Fach und woran können Sie sich erinnern?

Ergebnis: 4 antworten mit ,nein'. 10 konnten sich nicht mehr an die Inhalte erinnern. 13 nennen Beispiele (Stände: 6x; Karl der Große/ Staat und Kirche: 3x; Essen, Krankheiten, Kreuzzüge und Kurfürsten: 1x).

- Haben Sie in der Sekundarstufe II das Mittelalter behandelt? In welchem Fach und woran können Sie sich erinnern?

Ergebnis: 10 antworten mit, nein'; 3 sind sich nicht sicher, ob sie es vergessen haben; 5 haben mittelalterliche Themen behandelt, ohne sich erinnern zu können; 10 können sich an Inhalte erinnern, wobei nur Stände/ Gesellschaft und Tempelritter/Kreuzzüge mehrfach genannt werden; ansonsten jüdisches Leben und seine Bedrohung, Chlodwig, Pest und Hanse.

- Ist durch den Schulunterricht Ihr Interesse am Mittelalter eher verstärkt (6), eher nicht beeinflusst (16), eher weniger geworden (3)?

Ergebnis: Eine Person differenzierte: Sek I. verstärkte das Interesse, in Sek. II wurde es weniger.

- Haben Sie sich außerhalb des Schulunterrichts mit dem Mittelalter beschäftigt? Falls ja, wie und warum?

Ergebnis: 21 nennen vor allem historische Romane, Spiele und Serien.

- Wie ordnen Sie ihr Interesse an der mittelalterlichen Geschichte ein?

Ergebnis: Eher interessiert (15, 2 beantworteten die vorherige Frage mit ,nein'); weniger interessiert $(9,3$ beantworteten die vorherige Frage mit ,nein'); gar nicht interessiert (0) (2 ohne Antwort); kein Unterschied nach Geschlecht oder Alter erkennbar.

Semesterzahl Geschichte (sollte es sich um Ihr Zweit- oder Drittfach handeln, notieren Sie bitte ergänzend die Gesamtzahl der Semester): Korrespondiert mit Veranstaltungstyp und Alter

Studieren Sie auf Lehramt (22), Fach (4) oder anderes (0)?

Alter: 16-20 (9), 21-25 (15), älter als 25 (2)

Geschlecht: divers (0); männlich (14; 2 in der Übung); weiblich (12, 7 in der Übung) ${ }^{162}$

162 Der Diskussion im Kolloquium verdanke ich die Anregung für die Aufnahme einer weiteren Frage, die Hinweise zum Verhältnis von religiösem Wissen und Beurteilung der mittelalterlichen Kirche liefern könnte: Wurden Sie religiös sozialisiert? 